Article

\title{
Dating Oceanic Subduction in the Jurassic Bangong-Nujiang Oceanic Arc: A Zircon U-Pb Age and Lu-Hf Isotopes and Al-in-Hornblende Barometry Study of the Lameila Pluton in Western Tibet, China
}

\author{
De-Liang Liu®, Min Shi and Shao-Yong Jiang * \\ State Key Laboratory of Geological Processes and Mineral Resources, School of Earth Resources, \\ Collaborative Innovation Center for Exploration of Strategic Mineral Resources, China University of \\ Geosciences, Wuhan 430074, China; liudl@cug.edu.cn (D.-L.L.); shim@cug.edu.cn (M.S.) \\ * Correspondence: shyjiang@cug.edu.cn; Tel.: +86-027-6788-3058
}

Received: 20 October 2019; Accepted: 29 November 2019; Published: 4 December 2019

\begin{abstract}
The subduction and close of the Mesozoic Bangong-Nujiang Ocean (BNO) led to a collision of the Lhasa and Qiangtang blocks, which formed the backbone of the Tibetan Plateau (the largest and highest plateau on Earth). However, the detailed subduction processes (in particular, the oceanic subduction processes) within the $\mathrm{BNO}$ are still not clear. Here, we focus on the plutonic complex of the oceanic arc in the Bangong-Nujiang suture (BNS) and report field observations on zircon $\mathrm{U}-\mathrm{Pb}$ ages, $\mathrm{Lu}-\mathrm{Hf}$ isotopes, and the $\mathrm{Al}$-in-hornblende barometry of quartz diorites from the Lameila pluton in western Tibet. Zircon from the quartz diorites yielded a LA-ICP-MS U-Pb age of $164 \mathrm{Ma}$. The zircon showed very positive $\varepsilon_{\mathrm{Hf}}(\mathrm{t})$ values from 10.5 to 13.9 , suggesting the Lameila pluton was likely sourced from the depleted-mantle wedge, which is in contrast with contemporary (164-161 Ma) volcanic rocks in the region that had negative $\varepsilon_{\mathrm{Hf}}(\mathrm{t})$ values of -7.4 to -16.2 and a magma source from partial melting of subducted sediments. The Lameila pluton showed a temperature-corrected Al-in-hornblende pressure of $3.9 \pm 0.8 \mathrm{kbar}$, corresponding to an emplacement depth of $13 \pm 3 \mathrm{~km}$. Therefore, the thickness of the Jurassic oceanic arc crust must have doubled since the initial growth of the oceanic arc on the BNO crust, with a crustal thickness of $6.5 \mathrm{~km}$ during the Middle Jurassic. In combination with previous works on volcanic rocks, this study further supports a two-subduction zone model in association with the BNO during the Middle Jurassic, namely, a north-dipping BNO-Qiangtang subduction zone and an oceanic subduction zone within the BNO. The latter oceanic subduction zone produced the depleted-mantle-derived Lameila pluton and the subducted sediment-derived volcanic rocks in the fore arc.
\end{abstract}

Keywords: Al-in-hornblende; Lameila pluton; oceanic arc; Bangong-Nujiang; Tibet

\section{Introduction}

The Tibetan Plateau is the largest plateau on Earth, with an average elevation above $4 \mathrm{~km}$ [1] and crustal thickness of 60-80 $\mathrm{km}$ [2] over an area of 2.5 million $\mathrm{km}^{2}$. It was formed by the sequential amalgamation of continental or oceanic arc blocks (Figure 1) over several orogenic cycles since the Paleozoic, and further enhanced by the collision and continuing convergence of the India and Asia continents since the Cenozoic [3-5]. Knowledge of the pre-Cenozoic tectonics, particularly the Lhasa-Qiangtang collision that followed the closure of the Bangong-Nujiang Ocean (BNO), are critical in understanding the formation processes of the Tibetan Plateau. While much progress has been made recently in understanding the processes of the oceanic-continental subduction and the subsequent 
collision between the Lhasa and Qiangtang blocks in the past decades [6-16], the oceanic subduction process within the $\mathrm{BNO}$ is still unclear.

Evidence for the oceanic subduction and oceanic arc development come mainly from studies of volcanic rocks with boninitic geochemical characteristics. Zhang et al. [17] reported the occurrence of high-Mg quartz gabbro/diabase within the Bangong-Nujiang suture (BNS) near Dingqing, and proposed an oceanic subduction within the BNO. According to Shi et al. [18], the boninitic andesites in the BNS near Rutog record the fore arc volcanism associated with oceanic subduction. Recent studies on the 164-161 Ma high-Mg andesites from Shiquanhe [19] and Daruco [20] have suggested a Jurassic oceanic arc developed within the BNO. Apart from these significant findings, the configuration of this arc, such as its crustal thickness and the source(s) for the arc magmatism are still unclear.

In this work, we report a mineralogical and isotopic study on the plutonic part of the BNO oceanic arc in western Tibet. Field observations, zircon $\mathrm{U}-\mathrm{Pb}$ ages and $\mathrm{Lu}-\mathrm{Hf}$ isotopes, in combination with the Al-in-hornblende barometry of quartz diorites from the Lameila pluton, provide robust new evidence for the development and configuration of the Jurassic oceanic arc within the BNO.

\section{Geological Background}

\subsection{Tectonic Evolution of the Bangong-Nujiang Ocean}

The Mesozoic BNO separates the Qiangtang block to the north and the Lhasa block to the south (Figure 1). Rifting, which eventually led to formation of the oceanic basin, started at the Permian-Triassic boundary (Figure 2a). The start of rifting is evidenced by the initiation of separate sedimentary evolutions in the Qiangtang and Lhasa blocks, respectively, from the Triassic onward [21,22]. This is also supported by the oldest age (260-240 Ma) of mafic rocks from the ophiolites in the BNS [9,23,24]. The BNO rapidly developed and became a $5000 \pm 1020 \mathrm{~km}$ wide ocean by the Late Triassic, as recorded by the $45.1^{\circ} \pm 9.2^{\circ}$ paleolatitude gap between the Qiangtang and Lhasa blocks [25-27]. The subduction of the BNO beneath the Qiangtang block gave birth to the Jurassic-Cretaceous continental arc along the southern margin of the Qiangtang block (Figure 2b) [13,15,28,29], and caused ophiolite obduction near Dongqiao during the Middle Jurassic [30]. Synchronous to the oceanic-continental subduction, oceanic subduction within the BNO also commenced no later than the Middle Jurassic, and consequently a Jurassic oceanic arc was formed south of the Qiangtang continental arc (Figure 2b) [19,20,31,32]. As these subductions continued, the ocean basin narrowed rapidly. During the Middle Jurassic, the width of the BNO was approximately $2600 \pm 710 \mathrm{~km}\left(23.4^{\circ} \pm 6.4^{\circ}\right.$ paleolatitude gap between the Qiangtang and Lhasa blocks) [26].

Whether or not a south-dipping subduction of the BNO occurred is highly debatable (Figure 2c). The main evidence for a south-dipping subduction of the BNO is the occurrence of Early Cretaceous magmatic rocks with depleted isotopic characteristics in the northern margin of the Lhasa block. These magmatic rocks are suggested to be the result of the south-dipping BNO subduction $[7,33]$, however the first-order structures along the BNS do not support this inference [8,34,35]. Alternatively, the Cretaceous magmatic rocks in the northern margin of the Lhasa block could have been formed by the north-dipping subduction of the Indus-Yarlung Tethyan Ocean [36].

The BNO closed during the Early Cretaceous or Late Cretaceous, and the seawater became shallow such that the sedimentary facies changed from marine to non-marine between 125 and $118 \mathrm{Ma}$ [36]. The magmatism changed from low-temperature arc-type to high-temperature bimodal suites (high- $\mathrm{Nb}$ basalt and rhyolite with an age of approximately $90 \mathrm{Ma}$ ) locally along the BNS at the Early-Late Cretaceous boundary (Figure 2d) [37].

\subsection{Geology of the Study Area}

The study area is located near Shiquanhe in western Tibet (Figure 1). This area consists of three first-order tectonic units including the south margin of the Qiangtang block, the BNS, and the north margin of the Lhasa block (Figure 3). The Qiangtang block exposes Permian to Triassic bioclastic 
limestones intercalated with slates that were intruded by the Jurassic Larelaxin pluton with an age of 168-160 Ma [38,39]. This pluton is the westernmost part of the Jurassic-Cretaceous arc in the south margin of the Qiangtang block [29]. The BNS separates the Qiangtang block to the north and the Lhasa block to the south (Figure 1), and consists of two belts of ophiolitic mélange near Rutog (the west part of the Bangong-Nujiang ophiolite belt, BNOB) and Shiquanhe (the west part of the Shiquanhe-Nam Co ophiolite belt, SNOB) that were imbricated by south-directed thrusts $[8,35,40]$. Zircon U-Pb ages of the Rutog ophiolitic mélange span from 232 to $167 \mathrm{Ma}$ [31,41], and those of the Shiquanhe ophiolitic mélange range between 193 and 161 Ma [42]. Jurassic flysch crops out in the northern part of the study area, whereas Cretaceous clastic sediments bearing volcanic rocks and limestone crop out in the southern part of the study area. The northern margin of the Lhasa block exposes Permian-Triassic limestone interlayered with shale and the above-mentioned Cretaceous strata. The BNS and Lhasa block were intruded by Late Cretaceous granites.

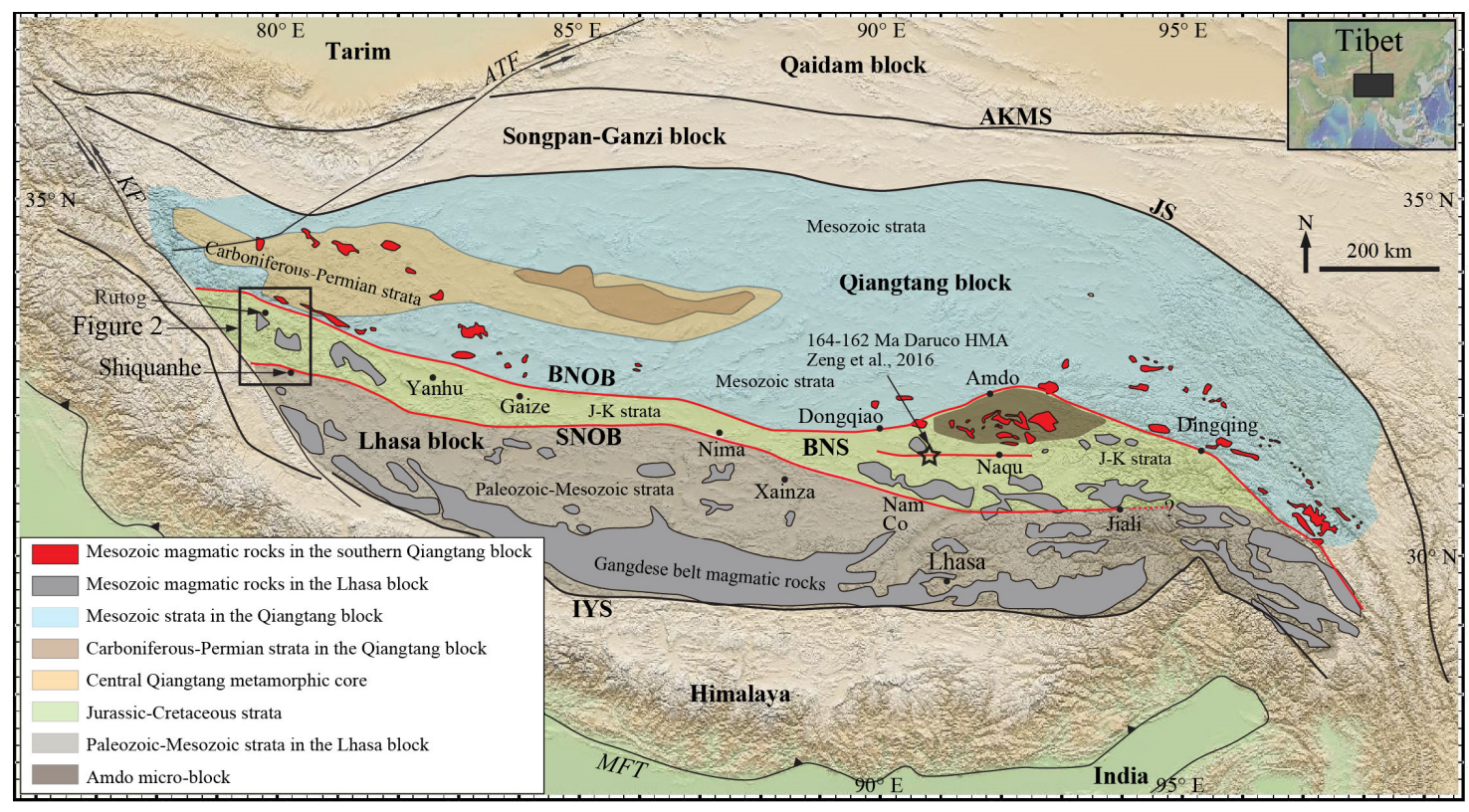

Figure 1. Geologic map of the Tibetan plateau. Base map is from GeoMapApp (www.geomapapp. org). Exposures of central Qiangtang metamorphic core, Carboniferous-Permian and Mesozoic strata in the Qiangtang block are modified after Kapp et al. [43], magmatic rocks in the southern Qiangtang block are modified after Liu et al. [15], and other geologic units are modified after Yin and Harrison [5]. Abbreviations: ATF $=$ Altyn Tagh fault, $\mathrm{KF}=$ Karakoram fault, MFT $=$ Main Frontal Thrust, IYS = Indus-Yarlung suture, BNS = Bangong-Nujiang suture, BNOB = Bangong-Nujiang ophiolite belt, SNOB = Shiquanhe-Nam Co ophiolite belt, JS = Jinsha suture, AKMS = Ayimaqin-Kunlun Mutztagh suture. Inset shows the location of Tibet. Black box shows the study area of this paper.

The boninitic andesites and 167 Ma supra-subduction zone (SSZ) type ophiolites [18,31] west of Rutog and the 161 Ma high-Mg andesites [44] north of Shiquanhe are the surface traces of the Jurassic oceanic arc within the BNS. The $164 \mathrm{Ma}$ Lameila pluton newly recognized in this work lies $20 \mathrm{~km}$ to the WWN of the Shiquanhe high-Mg andesites. This pluton trends WWN and is $20 \mathrm{~m}$ long and 1-3 km wide (Figure 3). It was covered by Jurassic flysch and Cretaceous sedimentary rocks. The Lameila pluton and Jurassic flysch were thrust over the Cretaceous strata by the north-dipping Jiagang thrust [8]. 
(a) P3-T1 (260-245 Ma): Initial rifting of the BNO

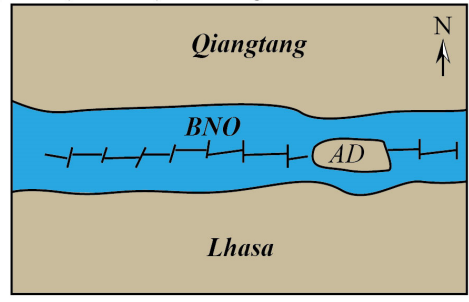

(c) J3-K1 (161-118 Ma): Continue subduction

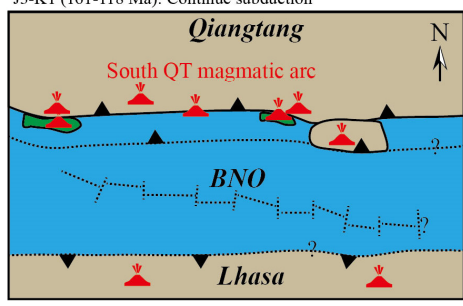

(b) J1-2 (202-161 Ma): Continental and oceanic arc development

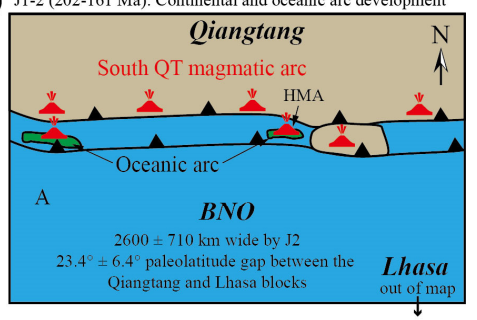

(d) K1-K2 (118-90 Ma): Lhasa-Qiangtang collision

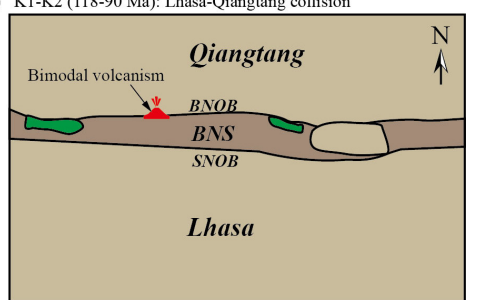

Figure 2. Simplified sketch map showing the possible evolution models of the Bangong-Nujiang Ocean. $\mathrm{AD}=$ Amdo micro-block; please see Figure 1 for other abbreviations. (a): Initial rifting of the Bangong-Nujiang Ocean at the Permian-Triassic boundary; (b): Continental and oceanic arc development during the Early and Middle Jurassic; (c): Continue subduction of the Bangong-Nujiang Ocean during the Middle Jurassic to Early Cretaceous; (d): The Lhasa-Qiangtang collision between 118 and $90 \mathrm{Ma}$.

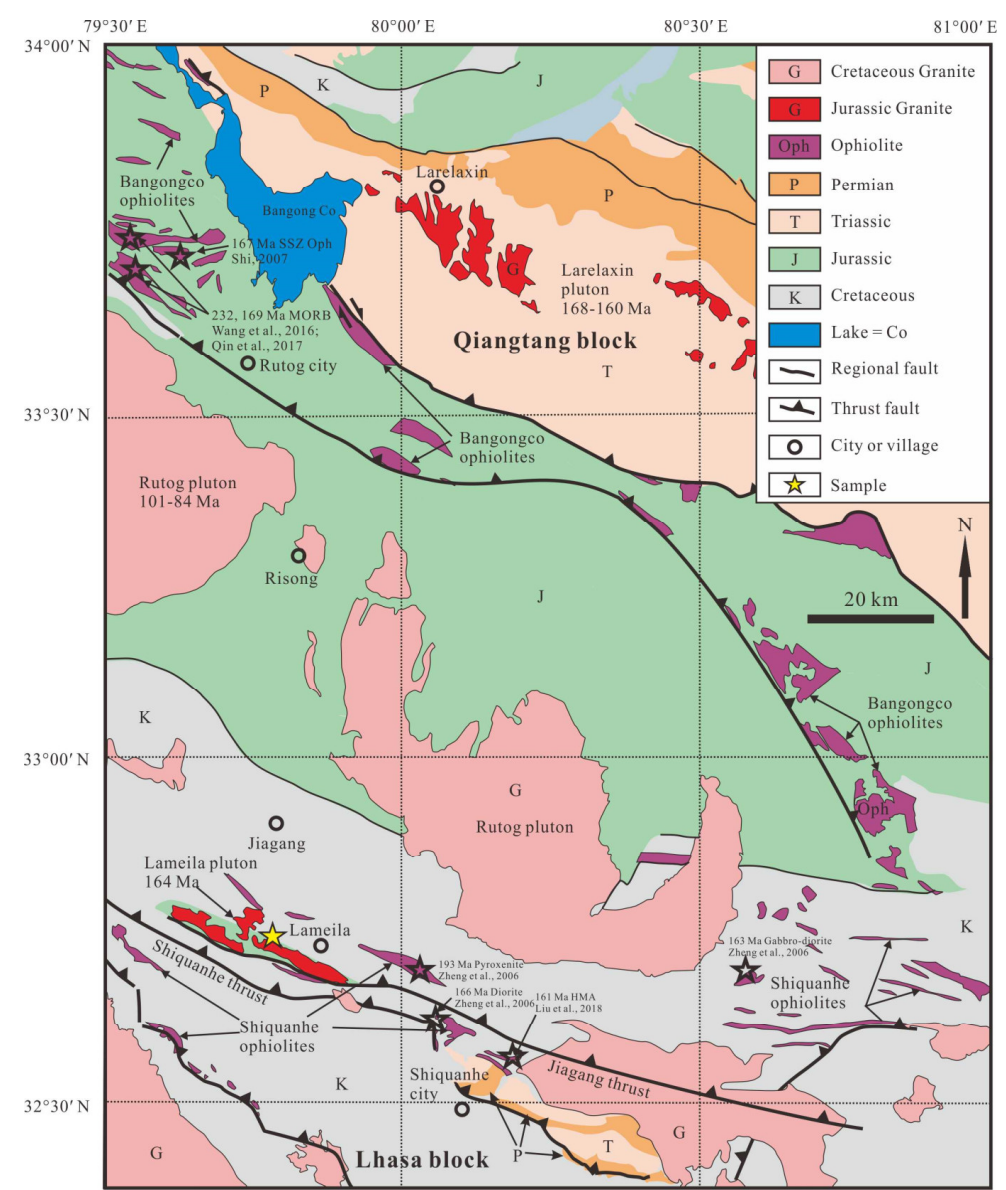

Figure 3. Geologic map of the Shiquanhe area modified after Liu et al. [39]. The sample location for this study is shown as a yellow star, and published ages of ophiolites and their locations are shown as unfilled stars. 


\section{Samples and Analytical Methods}

\subsection{Sampling and Petrography}

The Lameila pluton consisted mainly of middle- to fine-grained massive quartz diorites that were grayish white in color (Figure 4a). Dark-colored amphibole-rich xenoliths of diorites are in sharp contact with the host quartz diorites (Figure $4 \mathrm{~b}$ ). We collected one quartz diorite sample

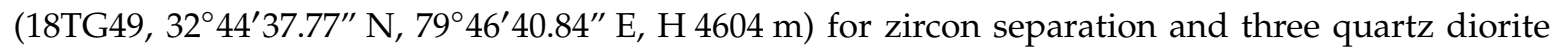
samples (18TG50, 18TG51 and 18TG52) for the Al-in-hornblende barometry study. The quartz diorites showed granitic texture, and consisted of plagioclase $(55 \%-60 \%)+$ hornblende $(10 \%-15 \%)+$ K-feldspar $(6 \%-10 \%)+$ quartz $(8 \%-10 \%)+$ biotite $(1 \%-2 \%)+$ magnetite + zircon. The plagioclase and hornblende were euhedral to subhedral, and most of the K-feldspar were subhedral; anhedral quartz occurred interstitial to the above minerals. Magnetite grains were enclosed in plagioclase, hornblende, and K-feldspar (Figure 4c). The hornblende grains were brown or dark green in color, and showed no signs of zoning patterns in both optical and electron backscatter electron images (Figure 4c,d). Zircon grains could locally be found enclosed in hornblende and plagioclase. Some hornblende crystals were partly altered to chlorite or biotite (Figure 4c). The above petrographic observations suggest that the Lameila quartz diorites contained the full buffering assemblages required for Al-in-hornblende barometry.
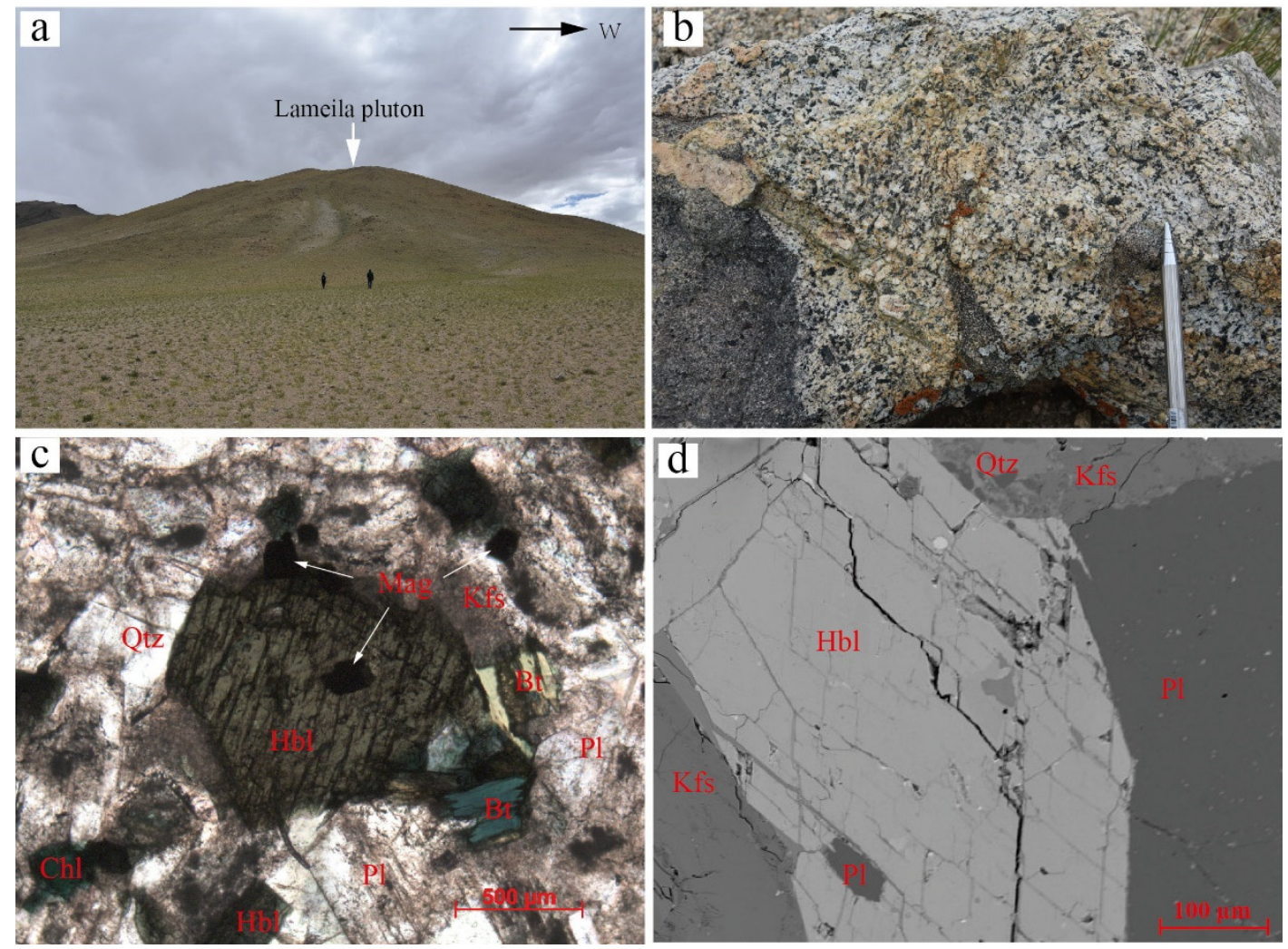

Figure 4. Field (a,b), photomicrograph (c), and backscattered electron (BSE) (d) images of the Lameila pluton. Abbreviations: $\mathrm{Qtz}=$ quartz, $\mathrm{Hbl}=$ hornblende, $\mathrm{Pl}=$ plagioclase, $\mathrm{Kfs}=\mathrm{K}$-feldspar, $\mathrm{Bt}=$ biotite, Mag = magnetite, $\mathrm{Chl}=$ chlorite.

\subsection{In-Situ LA-ICP-MS U-Pb Dating and Trace Elements of Zircon}

Zircon separation and cathodoluminescence (CL) images were carried out at the Guangzhou Tuoyan Testing Technology Co., Ltd. (Guangzhou, China). Approximately $5 \mathrm{~kg}$ of a rock sample was crushed and sieved for separating zircon grains using standard magnetic and heavy liquid separation procedures. About 150 grains were mounted in epoxy and polished to expose the center 
of crystals. In situ zircon U-Pb isotopic analyses were conducted using a RESOlution $193 \mathrm{~nm}$ ArF excimer laser (LA, Australian Scientific Instruments Pty Ltd., Canberra, Australia) coupled with a ThermoiCAP Qc Inductively Coupled Plasma-Mass Spectrometer (ICP-MS, Thermo Fisher Scientific Inc., Waltham, MA, USA) at the State Key Laboratory of Geological Processes and Mineral Resources, China University of Geosciences (Wuhan). The ablation system operated at a wavelength of $193 \mathrm{~nm}$ using a spot diameter of $33 \mu \mathrm{m}$ at a $10 \mathrm{~Hz}$ repetition rate for $40 \mathrm{~s}$, and ablation pits were about $20 \mu \mathrm{m}$ deep. Helium was used as carrier gas to transport the ablated materials to the ICP-MS. Each analysis consisted of a $15 \mathrm{~s}$ background acquisition, $40 \mathrm{~s}$ sample data acquisition, and $45 \mathrm{~s}$ washout delay at the end. Every 10 sample analyses were followed by analysis of one glass standard (NIST 612) and two zircon standards 91500 [45]. Analytical results were calculated using ICPMSDataCal software (Version number, Liu et al., Wuhan, China) [46]. Concordia U-Pb diagrams and weighted mean ${ }^{206} \mathrm{~Pb} /{ }^{238} \mathrm{U}$ age calculations were made using Isoplot v2.3 (Berkeley Geochronological Center, Berkeley, CA, USA) [47].

\subsection{In-Situ LA-MC-ICP-MS Lu-Hf Isotopes of Zircon}

In situ zircon Lu-Hf isotope analyses were carried out using the RESOlution-S155 193 nm ArF excimer laser, which was attached to a Nu Plasma II MC-ICP-MS at the State Key Laboratory of Geological Processes and Mineral Resources, China University of Geosciences (Wuhan). A stationary spot was used for the present analyses, with a beam diameter of $33 \mu \mathrm{m}$. Helium was used as the carrier gas to transport the ablated materials to the MC-ICP-MS. Atomic masses of 172, 173, 175-180, and 182 were simultaneously measured in static-collection mode. The ${ }^{176} \mathrm{Lu} /{ }^{175} \mathrm{Lu}=0.02655$ and ${ }^{176} \mathrm{Yb} /{ }^{173} \mathrm{Yb}=$ 0.7965 ratios were used to correct the isobaric interferences of ${ }^{176} \mathrm{Lu}$ and ${ }^{176} \mathrm{Yb}$ on ${ }^{176} \mathrm{Hf}$, respectively [48]. Penglai zircon was selected as the standard during the analysis, whose analytical results $\left({ }^{176} \mathrm{Hf} /{ }^{177} \mathrm{Hf}\right.$ $=0.282896 \pm 38$, mean square weighted deviation $(\mathrm{MSWD})=0.89, \mathrm{n}=25$ ) during our one-day analyses were in good agreement with the published data within error $(0.282906 \pm 0.000010)$ [49]. To calculate the $\varepsilon_{\mathrm{Hf}}(\mathrm{t})$ values, we adopted a decay constant for ${ }^{176} \mathrm{Lu}$ of $1.867 \times 10^{-11}$ year $^{-1}$ [50] and chondritic present-day values of ${ }^{176} \mathrm{Lu} /{ }^{177} \mathrm{Hf}(0.0336)$ and ${ }^{176} \mathrm{Hf} /{ }^{177} \mathrm{Hf}(0.282785)$ [51]. Depleted-mantle Hf model ages $\left(\mathrm{T}_{\mathrm{DM}}\right)$ were calculated using the measured ${ }^{176} \mathrm{Lu} /{ }^{177} \mathrm{Hf}$ ratios of zircon, assuming that the depleted-mantle reservoir had a ${ }^{176} \mathrm{Hf} /{ }^{177} \mathrm{Hf}$ of 0.283250 at present day, with a ${ }^{176} \mathrm{Lu} /{ }^{177} \mathrm{Hf}$ value of 0.0384 [52]. The mantle extraction model age $\left(\mathrm{T}_{\mathrm{DM}}{ }^{\mathrm{C}}\right)$ for the source rocks of the magmas was calculated by projecting initial ${ }^{176} \mathrm{Hf} /{ }^{177} \mathrm{Hf}$ ratios of the zircons to the depleted-mantle model growth line using a mean ${ }^{176} \mathrm{Lu} /{ }^{177} \mathrm{Hf}$ value $(0.015)$ for average continental crust [53].

\subsection{EPMA Amphibole Chemistry}

Mineral compositions were determined at the State Key Laboratory of Geological Processes and Mineral Resources, China University of Geosciences (Wuhan), with a JEOL JXA-8100 Electron Probe Micro Analyzer (EPMA, JEOL Ltd., Tokyo, Japan) equipped with four wavelength-dispersive spectrometers (WDSs). The samples were coated with a thin conductive carbon film prior to analysis. The precautions suggested by Zhang and Yang [54] were used to minimize the difference of carbon film thickness between samples and standards and obtain an approximately uniform $20 \mathrm{~nm}$ coating. During the analysis, an accelerating voltage of $15 \mathrm{kV}$, a beam current of $20 \mathrm{nA}$ and a 1- $\mu \mathrm{m}$ spot size were used to analyze the minerals. Data were corrected online using a modified ZAF (atomic number, absorption, fluorescence) correction procedure. The peak counting time was $10 \mathrm{~s}$ for $\mathrm{Na}, \mathrm{Mg}, \mathrm{Al}, \mathrm{Si}, \mathrm{K}$, $\mathrm{Ca}$, and Fe, and $20 \mathrm{~s}$ for $\mathrm{Ti}$ and $\mathrm{Mn}$. The background counting time was one-half of the peak counting time on the high- and low-energy background positions. The following standards were used: sanidine $(\mathrm{K})$, pyropegarnet $(\mathrm{Fe}, \mathrm{Al})$, diopsode $(\mathrm{Ca}, \mathrm{Mg})$, jadeite $(\mathrm{Na})$, rhodonite $(\mathrm{Mn})$, olivine (Si), and rutile (Ti). Precision of the EPMA analysis was calculated from counting statistics and was generally better than $\pm 1 \%$ for contents of $>10 \mathrm{wt} \%$, and better than $\pm 5 \%$ for contents of $>0.5 \mathrm{wt} \%$. 


\section{Results}

\subsection{Zircon $\mathrm{U}-\mathrm{Pb}$ Geochronology and Trace Elements}

Zircon grains in sample 18TG49 from the Lameila pluton were euhedral, with sizes that were 100-200 $\mu \mathrm{m}$ long and 80-120 $\mu \mathrm{m}$ wide. They showed oscillatory zoning patterns (Figure 5), and had low Th (68-318 ppm) and U (129-460 ppm) concentrations with high Th-U ratios (0.50-0.72). The U-Pb geochronological and trace element data are listed in Tables 1 and 2. Twenty-seven of the 30 total analyses yielded concordant ${ }^{206} \mathrm{~Pb} /{ }^{238} \mathrm{U}$ and ${ }^{207} \mathrm{~Pb} /{ }^{235} \mathrm{U}$ ages, with ${ }^{206} \mathrm{~Pb} /{ }^{238} \mathrm{U}$ ages between 158 and $169 \mathrm{Ma}$. The weighted mean age was $164.0 \pm 1.1 \mathrm{Ma}(\mathrm{n}=27, \mathrm{MSWD}=1.3$, Figure 6a,b). The oscillatory zoning patterns, Th and U concentrations, and typical magmatic zircon rare earth element (REE) patterns (Figure 6c) imply that this age represents the time of magma emplacement.

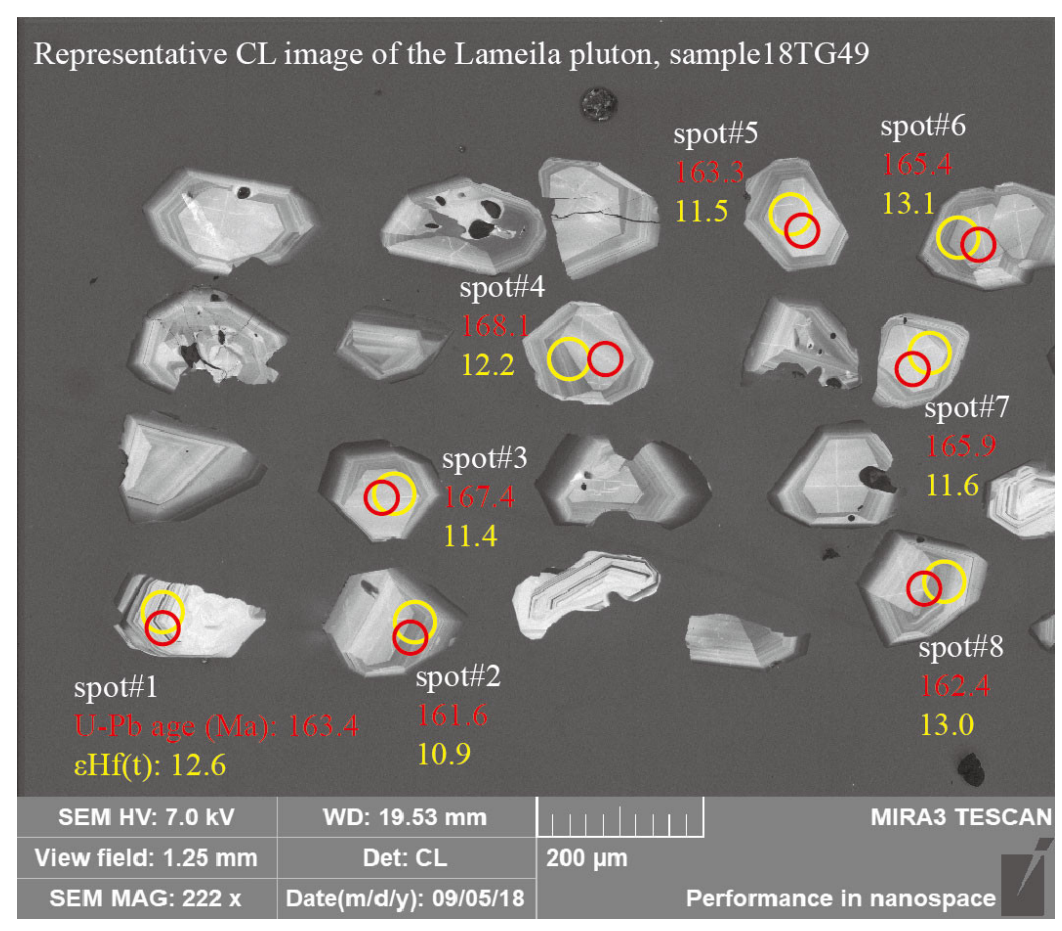

Figure 5. Cathodoluminescence (CL) image of zircons from the Lameila pluton. Red and yellow circles represent analytical spots for LA-ICP-MS zircon U-Pb and LA-MC-ICP-MS zircon Lu-Hf isotope measurements, and the analytical data are also shown. 
Table 1. LA-ICP-MS zircon U-Pb dating for the Lameila pluton.

\begin{tabular}{|c|c|c|c|c|c|c|c|c|c|c|c|c|c|c|c|c|}
\hline \multirow[b]{2}{*}{ Analysis } & \multirow[b]{2}{*}{$\begin{array}{c}\text { Th } \\
\text { (ppm) }\end{array}$} & \multirow[b]{2}{*}{$\underset{(\mathrm{ppm})}{\mathrm{U}}$} & \multirow[b]{2}{*}{$\mathrm{Th} / \mathrm{U}$} & \multicolumn{6}{|c|}{ Isotope Ratios } & \multicolumn{6}{|c|}{ Isotope Ages (Ma) } & \multirow[b]{2}{*}{$\begin{array}{c}\text { Discordant } \\
\quad(\%)\end{array}$} \\
\hline & & & & $\begin{array}{l}{ }^{207} \mathrm{~Pb} / \\
{ }^{206} \mathrm{~Pb}\end{array}$ & 1s & $\begin{array}{l}{ }^{207} \mathrm{~Pb} / \\
{ }^{235} \mathrm{U}\end{array}$ & 1s & $\begin{array}{l}{ }^{206} \mathrm{~Pb} / \\
{ }^{238} \mathrm{U}\end{array}$ & 1s & $\begin{array}{l}{ }^{207} \mathrm{~Pb} / \\
{ }^{206} \mathrm{~Pb}\end{array}$ & 1s & $\begin{array}{l}{ }^{207} \mathrm{~Pb} / \\
{ }^{235} \mathrm{U}\end{array}$ & $1 s$ & $\begin{array}{l}{ }^{206} \mathrm{~Pb} / \\
{ }^{238} \mathrm{U}\end{array}$ & 1s & \\
\hline TG-49-1 & 77.6 & 131 & 0.59 & 0.05031 & 0.00381 & 0.17434 & 0.01198 & 0.02568 & 0.00052 & 209.3 & 171.3 & 163.2 & 10.4 & 163.4 & 3.3 & $99 \%$ \\
\hline TG-49-2 & 164 & 278 & 0.59 & 0.05090 & 0.00265 & 0.17982 & 0.00949 & 0.02539 & 0.00039 & 235.3 & 115.7 & 167.9 & 8.2 & 161.6 & 2.5 & $96 \%$ \\
\hline TG-49-3 & 83.8 & 155 & 0.54 & 0.04984 & 0.00328 & 0.17730 & 0.01076 & 0.02630 & 0.00049 & 187.1 & 155.5 & 165.7 & 9.3 & 167.4 & 3.1 & $99 \%$ \\
\hline TG-49-4 & 89.7 & 163 & 0.55 & 0.04776 & 0.00348 & 0.17494 & 0.01119 & 0.02642 & 0.00044 & 87.1 & 162.9 & 163.7 & 9.7 & 168.1 & 2.8 & $97 \%$ \\
\hline TG-49-5 & 117 & 191 & 0.61 & 0.04956 & 0.00300 & 0.17455 & 0.00979 & 0.02566 & 0.00038 & 176.0 & 137.9 & 163.4 & 8.5 & 163.3 & 2.4 & $99 \%$ \\
\hline TG-49-6 & 156 & 224 & 0.70 & 0.04879 & 0.00297 & 0.17458 & 0.01024 & 0.02600 & 0.00039 & 200.1 & 75.9 & 163.4 & 8.9 & 165.4 & 2.5 & $98 \%$ \\
\hline TG-49-7 & 67.5 & 135 & 0.50 & 0.04839 & 0.00368 & 0.17626 & 0.01280 & 0.02607 & 0.00044 & 116.8 & 170.3 & 164.8 & 11.0 & 165.9 & 2.8 & $99 \%$ \\
\hline TG-49-8 & 114 & 195 & 0.58 & 0.04969 & 0.00300 & 0.17621 & 0.01065 & 0.02547 & 0.00042 & 189.0 & 142.6 & 164.8 & 9.2 & 162.2 & 2.6 & $98 \%$ \\
\hline TG-49-10 & 136 & 240 & 0.57 & 0.05038 & 0.00303 & 0.18133 & 0.01004 & 0.02624 & 0.00036 & 213.0 & 140.7 & 169.2 & 8.6 & 167.0 & 2.3 & $98 \%$ \\
\hline TG-49-11 & 142 & 263 & 0.54 & 0.04984 & 0.00329 & 0.17727 & 0.01167 & 0.02548 & 0.00038 & 187.1 & 155.5 & 165.7 & 10.1 & 162.2 & 2.4 & $97 \%$ \\
\hline TG-49-12 & 221 & 326 & 0.68 & 0.05038 & 0.00270 & 0.17300 & 0.00914 & 0.02486 & 0.00034 & 213.0 & 124.1 & 162.0 & 7.9 & 158.3 & 2.2 & $97 \%$ \\
\hline TG-49-13 & 82.6 & 144 & 0.57 & 0.05000 & 0.00400 & 0.17835 & 0.01272 & 0.02614 & 0.00051 & 194.5 & 177.8 & 166.6 & 11.0 & 166.4 & 3.2 & $99 \%$ \\
\hline TG-49-14 & 318 & 439 & 0.72 & 0.04824 & 0.00238 & 0.17222 & 0.00882 & 0.02548 & 0.00032 & 109.4 & 124.1 & 161.3 & 7.6 & 162.2 & 2.0 & $99 \%$ \\
\hline TG-49-15 & 128 & 225 & 0.57 & 0.04745 & 0.00294 & 0.16620 & 0.00978 & 0.02534 & 0.00042 & 77.9 & 135.2 & 156.1 & 8.5 & 161.3 & 2.7 & $96 \%$ \\
\hline TG-49-16 & 125 & 215 & 0.58 & 0.05128 & 0.00385 & 0.18500 & 0.01305 & 0.02612 & 0.00041 & 253.8 & 169.4 & 172.4 & 11.2 & 166.2 & 2.6 & $96 \%$ \\
\hline TG-49-17 & 217 & 378 & 0.57 & 0.04843 & 0.00276 & 0.17221 & 0.00931 & 0.02569 & 0.00035 & 120.5 & 129.6 & 161.3 & 8.1 & 163.5 & 2.2 & $98 \%$ \\
\hline TG-49-18 & 137 & 237 & 0.58 & 0.04818 & 0.00315 & 0.17349 & 0.01014 & 0.02604 & 0.00041 & 109.4 & 148.1 & 162.4 & 8.8 & 165.7 & 2.6 & $98 \%$ \\
\hline TG-49-19 & 217 & 324 & 0.67 & 0.04392 & 0.00267 & 0.16182 & 0.00982 & 0.02637 & 0.00042 & error & error & 152.3 & 8.6 & 167.8 & 2.6 & $90 \%$ \\
\hline TG-49-20 & 199 & 352 & 0.57 & 0.04723 & 0.00283 & 0.16394 & 0.00929 & 0.02495 & 0.00040 & 61.2 & 137.0 & 154.1 & 8.1 & 158.8 & 2.5 & $97 \%$ \\
\hline TG-49-21 & 67.6 & 129 & 0.52 & 0.04950 & 0.00406 & 0.17970 & 0.01408 & 0.02575 & 0.00064 & 172.3 & 181.5 & 167.8 & 12.1 & 163.9 & 4.0 & $97 \%$ \\
\hline TG-49-22 & 311 & 459 & 0.68 & 0.04772 & 0.00219 & 0.16739 & 0.00723 & 0.02535 & 0.00037 & 87.1 & 103.7 & 157.2 & 6.3 & 161.4 & 2.3 & $97 \%$ \\
\hline TG-49-23 & 213 & 350 & 0.61 & 0.04815 & 0.00248 & 0.17252 & 0.00879 & 0.02579 & 0.00041 & 105.6 & 127.8 & 161.6 & 7.6 & 164.2 & 2.6 & $98 \%$ \\
\hline TG-49-24 & 257 & 379 & 0.68 & 0.04773 & 0.00263 & 0.16848 & 0.00896 & 0.02557 & 0.00034 & 87.1 & 125.9 & 158.1 & 7.8 & 162.8 & 2.1 & $97 \%$ \\
\hline TG-49-27 & 103 & 176 & 0.58 & 0.05028 & 0.00381 & 0.18149 & 0.01243 & 0.02619 & 0.00046 & 209.3 & 171.3 & 169.3 & 10.7 & 166.6 & 2.9 & $98 \%$ \\
\hline TG-49-28 & 219 & 331 & 0.66 & 0.04438 & 0.00236 & 0.15994 & 0.00824 & 0.02607 & 0.00037 & error & error & 150.6 & 7.2 & 165.9 & 2.3 & $90 \%$ \\
\hline TG-49-29 & 123 & 217 & 0.57 & 0.04769 & 0.00320 & 0.17719 & 0.01204 & 0.02664 & 0.00039 & 83.4 & 151.8 & 165.6 & 10.4 & 169.5 & 2.5 & $97 \%$ \\
\hline TG-49-30 & 186 & 302 & 0.62 & 0.04790 & 0.00280 & 0.17132 & 0.00971 & 0.02591 & 0.00041 & 94.5 & 133.3 & 160.6 & 8.4 & 164.9 & 2.6 & $97 \%$ \\
\hline
\end{tabular}


Table 2. Zircon trace element results for the Lameila pluton.

\begin{tabular}{|c|c|c|c|c|c|c|c|c|c|c|c|c|c|c|c|}
\hline Spot & $\mathrm{Ti}$ & $\mathbf{L a}$ & $\mathrm{Ce}$ & Pr & $\mathrm{Nd}$ & $\mathrm{Sm}$ & $\mathbf{E u}$ & Gd & $\mathrm{Tb}$ & Dy & Ho & Er & Tm & $\mathrm{Yb}$ & $\mathbf{L u}$ \\
\hline $\mathrm{T}$ & 589 & 05 & 5.1 & & 066 & I & 3 & 3.62 & 3 & 1 & 5 & 3.87 & 14.69 & 61.1 & 40.03 \\
\hline & 7.02 & 0.01 & 7.89 & 02 & 51 & 0.96 & 69 & 8.75 & .06 & & 30.52 & 170 & 40.01 & & 101 \\
\hline TG-49-3 & 5.87 & 0.01 & 4.54 & 0.02 & 0.4 & 0.98 & 0.59 & 6.56 & 2.54 & 39.26 & 18.04 & 103 & 25.39 & 277.0 & 70.97 \\
\hline TG-49-4 & 1.56 & 0 & 4.63 & 0.01 & 0.47 & 0.93 & 0.53 & 6.44 & 2.65 & 41.27 & 18.82 & 108 & 26.52 & 286 & 73.25 \\
\hline TG-49-5 & 1.81 & 0 & 5.68 & 0.04 & 0.49 & 1.02 & 0.64 & 7.64 & 3.4 & 50.83 & 22.36 & 124 & 29.57 & 314 & 77.54 \\
\hline TG-49-6 & 3.96 & 0.01 & 6.75 & 0.05 & 0.82 & 1.42 & 0.89 & 10.54 & 4.07 & & & & & 363 & 89.11 \\
\hline TG-49-7 & 3.07 & 0.01 & 3.87 & 0 & 0.32 & 0.78 & 0.45 & & 2.09 & & & & & & 60.21 \\
\hline & 8.04 & 0 & 5. & 0 & 0. & & & & & & & & & & \\
\hline & 2. & 0 & & & & & & & & & & & & & \\
\hline & & U & & & & & & & & & & & & & \\
\hline & 7 & & 9. & & & & & & & & & & & & 115 \\
\hline & 4. & & 4.9 & & 0.3 & 1. & 0.3 & & 2.42 & 38.97 & 17. & & 6 & 286 & 71.75 \\
\hline & 8.23 & & 11.9 & & 0.69 & & & & 6.81 & & & & 58.79 & 609 & 143 \\
\hline & 7.23 & & & 0. & 0.42 & 1. & 0.7 & . & 3.42 & & 25.6 & 15 & 36.42 & 394 & 97.83 \\
\hline 16 & 2.09 & 2 & 6.39 & 0.04 & 0.59 & 1.26 & 0.74 & 8.7 & 3.58 & 7 & 24.96 & 14 & 34.48 & 378 & 95.16 \\
\hline & 5.06 & 0.01 & 10.24 & 0.03 & 0.67 & 1.28 & 0.76 & 10.62 & 5.09 & & 39.26 & 224 & & 554 & 134 \\
\hline & 11.3 & 6.15 & 20.91 & 1.4 & 6 & 2.1 & 0.94 & 9.67 & 3.71 & & 27.09 & 15 & 37.35 & 404 & 101 \\
\hline 49-19 & 3.06 & 0.02 & 9.2 & 0.03 & 0.69 & 1.57 & 0.95 & 12.29 & 5.1 & 80.69 & 36.14 & 209 & 49.3 & 527 & 130 \\
\hline TG-49-20 & 7.06 & 0.01 & 9.58 & 0.03 & 0.63 & 1.38 & 0.92 & 11.54 & 5.2 & 83.23 & 37.23 & 214 & 50.63 & 545 & 132 \\
\hline TG-49-21 & 5.58 & 0.02 & 4.33 & 0.02 & 0.42 & 0.73 & 0.39 & 5.6 & 2.43 & 35.71 & 16.05 & 94.67 & 24.02 & 268 & 68.25 \\
\hline TG-49-22 & 8.62 & 0.01 & 13.4 & 0.05 & 0.72 & 1.36 & 0.98 & 13.83 & 6.78 & 113 & 50.14 & 276 & 63.66 & 656 & 156 \\
\hline TG-49-23 & 7.88 & 0.01 & 10.16 & 0.04 & 0.6 & 1.1 & 0.77 & 11.21 & 5.09 & 84.17 & 37.73 & 21 & 49.78 & 517 & 126 \\
\hline TG-49-24 & 8.28 & 0 & 11 & 0.03 & 0.6 & & 0.89 & 12.78 & 5.72 & 94.2 & 41.68 & 22 & 52.92 & 548 & 132 \\
\hline TG-4 & 10.25 & 0.0 & & 0.04 & 0.4 & & 0. & & 2.8 & & 6 & & 27.12 & 287 & 72.33 \\
\hline TG & & & & 0.0 & 0.7 & & & 11.44 & & & & & 47.97 & 493 & 118 \\
\hline & & & & & & & & & & & & & & & 89.29 \\
\hline TG-49-30 & 5.14 & 0.01 & 8.73 & 0.02 & 0.49 & 1.11 & 0.63 & 9.91 & 4.44 & 73.33 & 32.92 & 181 & 42.8 & 446 & 108 \\
\hline
\end{tabular}



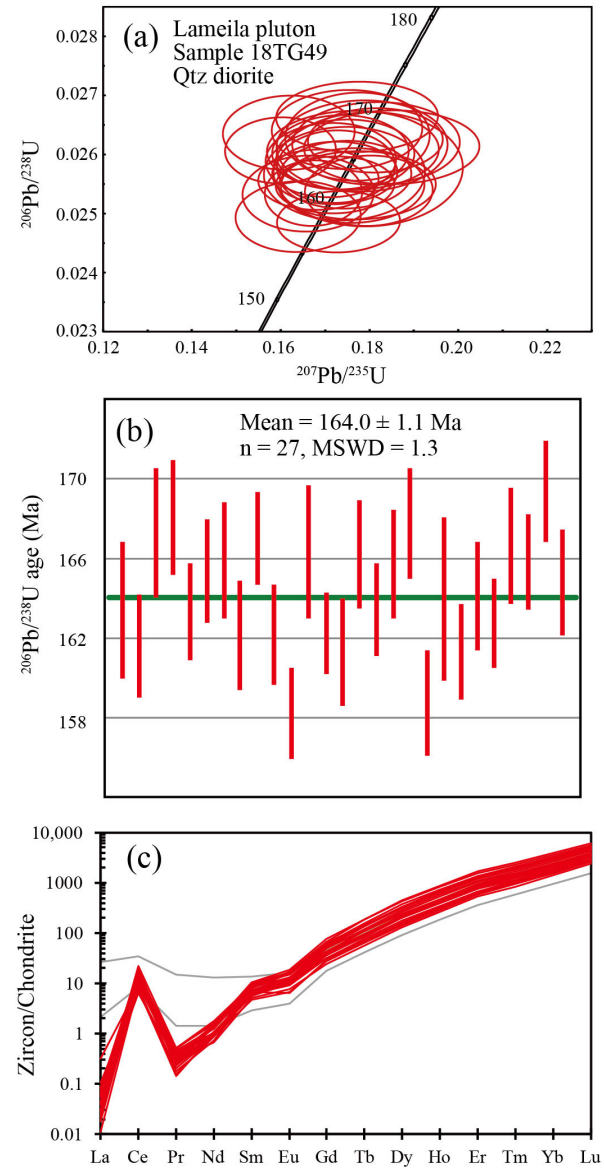

Figure 6. Zircon $\mathrm{U}-\mathrm{Pb}$ ages $(\mathbf{a}, \mathbf{b})$ and chondrite-normalized [55] rare earth element patterns (c). The error bars in (b) refer to one sigma. The two outliers (spots TG-49-1 and TG-49-18 in Table 2) shown as gray lines in (c) are likely the result of tiny inclusions of mineral or fluid with slightly higher light rare earth elements (LREE) than the host zircon.

\subsection{In-Situ Zircon Lu-Hf Isotopes}

Zircons from the Lameila pluton had ${ }^{176} \mathrm{Hf} /{ }^{177} \mathrm{Hf}$ ratios ranging from 0.282972 to 0.283068 (Table 3), the initial ${ }^{176} \mathrm{Hf} /{ }^{177} \mathrm{Hf}$ ratios calculated using the corresponding zircon ages were $0.282966-0.283063$, and the $\varepsilon_{\mathrm{Hf}}(\mathrm{t})$ values ranged from 10.5 to 13.9 (Figure 7 ). These had very young $\mathrm{T}_{\mathrm{DM}}$ ages of $270-410 \mathrm{Ma}$, with an average of $340 \mathrm{Ma}$. The $\mathrm{T}_{\mathrm{DM}}{ }^{\mathrm{C}}$ ages were between 320 and $540 \mathrm{Ma}$, with an average age of $440 \mathrm{Ma}$.

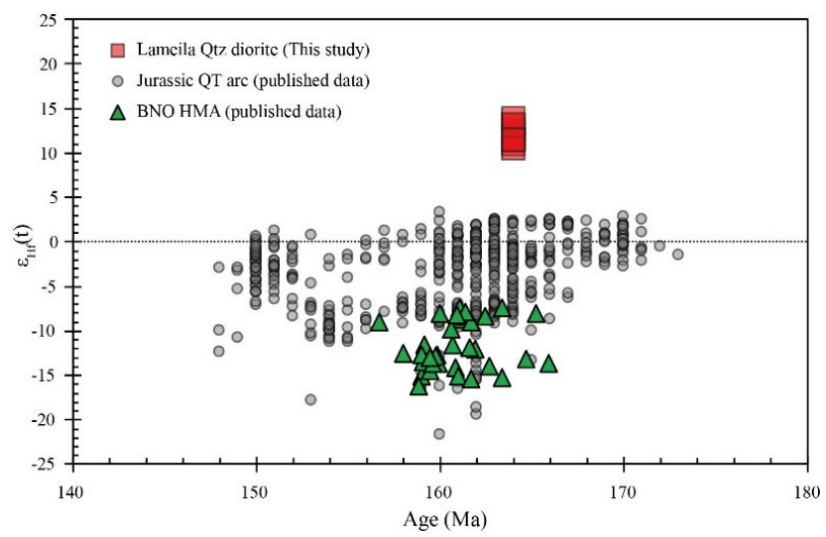

Figure 7. Plot of $\varepsilon_{\mathrm{Hf}}(\mathrm{t})$ versus age for the Lameila pluton. Data for the Jurassic Qiangtang arc are from Liu et al. [15]; data for the BNO high-Mg andesite are from Liu et al. [19] and Zeng et al. [20]. 
Table 3. LA-MC-ICP-MS zircon Lu-Hf isotopes of the Lameila pluton.

\begin{tabular}{|c|c|c|c|c|c|c|c|c|c|c|c|}
\hline Spot & Age (Ma) & $\begin{array}{l}176 \mathrm{Hf} / \\
{ }^{177} \mathrm{Hf}\end{array}$ & $2 \sigma$ & $\begin{array}{l}176 \mathrm{Yb} / \\
{ }^{177} \mathrm{Hf}\end{array}$ & $2 \sigma$ & $\begin{array}{l}{ }^{176} \mathrm{Lu} / \\
{ }^{177} \mathrm{Hf}\end{array}$ & $2 \sigma$ & $\begin{array}{l}\left({ }^{176} \mathrm{Hf} /\right. \\
\left.{ }^{177} \mathrm{Hf}\right)_{\mathrm{i}}\end{array}$ & $\varepsilon_{\mathrm{Hf}}(\mathrm{t})$ & $\mathrm{T}_{\mathrm{DM}}(\mathrm{Ma})$ & $\begin{array}{c}\mathrm{T}_{\mathrm{DM}}^{\mathrm{C}} \\
\text { (Ma) }\end{array}$ \\
\hline 18TG-49-1 & 164 & 0.283028 & 0.000013 & 0.006345 & 0.000118 & 0.000327 & 0.000006 & 0.283027 & 12.6 & 312 & 405 \\
\hline 18TG-49-2 & 164 & 0.282985 & 0.000020 & 0.035704 & 0.000358 & 0.001775 & 0.000015 & 0.282980 & 10.9 & 386 & 512 \\
\hline 18TG-49-3 & 164 & 0.282998 & 0.000016 & 0.033158 & 0.000295 & 0.001620 & 0.000015 & 0.282993 & 11.4 & 367 & 482 \\
\hline 18TG-49-4 & 164 & 0.283019 & 0.000015 & 0.032713 & 0.000422 & 0.001657 & 0.000020 & 0.283014 & 12.2 & 336 & 433 \\
\hline 18TG-49-5 & 164 & 0.283000 & 0.000016 & 0.031823 & 0.000122 & 0.001578 & 0.000010 & 0.282995 & 11.5 & 363 & 477 \\
\hline 18TG-49-6 & 164 & 0.283047 & 0.000014 & 0.035129 & 0.000156 & 0.001746 & 0.000008 & 0.283042 & 13.1 & 296 & 371 \\
\hline $18 T G-49-7$ & 164 & 0.283001 & 0.000016 & 0.024398 & 0.000150 & 0.001198 & 0.000005 & 0.282997 & 11.6 & 358 & 473 \\
\hline 18TG-49-8 & 164 & 0.283042 & 0.000017 & 0.032354 & 0.000163 & 0.001599 & 0.000013 & 0.283037 & 13.0 & 302 & 381 \\
\hline 18TG-49-10 & 164 & 0.282972 & 0.000015 & 0.039386 & 0.000686 & 0.001887 & 0.000033 & 0.282966 & 10.5 & 407 & 542 \\
\hline 18TG-49-11 & 164 & 0.283068 & 0.000016 & 0.037275 & 0.000264 & 0.001773 & 0.000014 & 0.283063 & 13.9 & 265 & 323 \\
\hline 18TG-49-12 & 164 & 0.282999 & 0.000018 & 0.030743 & 0.000285 & 0.001501 & 0.000012 & 0.282994 & 11.5 & 364 & 479 \\
\hline
\end{tabular}




\subsection{Amphibole Chemistry and Stoichiometry}

Results of the EPMA analyses for amphibole chemistry are shown in Table 4. Locations for EPMA analysis were near the margins in contact with quartz or K-feldspar. The amphibole compositions of the three quartz diorite samples were broadly the same; therefore, we present the major element compositions and assess the dominance of atomic exchange reactions by treating all of the amphibole crystals as one population. All the amphiboles from the Lameila pluton displayed a narrow compositional range in $\mathrm{SiO}_{2}(45.33-49.84 \mathrm{wt} \%$, average $=47.13 \mathrm{wt} \%)$ and $\mathrm{CaO}(10.30-11.88 \mathrm{wt} \%$, average $=11.23 \mathrm{wt} \%$ ). Their $\mathrm{Al}_{2} \mathrm{O}_{3}$ contents were uniform and low, ranging between 6.32 and $8.81 \mathrm{wt} \%$ (average $=7.86 \mathrm{wt} \%)$, there are some variation for $\mathrm{MgO}(11.81-15.41 \mathrm{wt} \%$, average $=12.96 \mathrm{wt} \%$ ) and total $\mathrm{FeO}(12.52-17.25 \mathrm{wt} \%$, average $=15.23 \mathrm{wt} \%)$ compositions (Table 4).

Table 4. EPMA hornblende compositions and Al-in-hornblende pressure results of the Lameila pluton.

\begin{tabular}{|c|c|c|c|c|c|c|c|c|c|c|c|}
\hline Spot & TG50-1 & TG50-2 & TG50-3 & TG50-4 & TG50-5 & TG50-6 & TG50-7 & TG50-8 & TG51-1 & TG51-2 & TG51-3 \\
\hline $\mathrm{TiO}_{2}$ & 0.57 & 0.63 & 1.10 & 0.63 & 0.68 & 0.70 & 0.76 & 0.63 & 0.56 & 0.59 & 0.74 \\
\hline $\mathrm{FeO}_{\text {total }}$ & 17.00 & 15.13 & 13.58 & 15.87 & 15.86 & 16.19 & 17.25 & 16.26 & 15.66 & 16.23 & 12.52 \\
\hline $\mathrm{MnO}$ & 0.71 & 0.69 & 0.45 & 0.72 & 0.73 & 0.78 & 0.81 & 0.72 & 0.59 & 0.52 & 0.43 \\
\hline $\mathrm{MgO}$ & 11.82 & 12.65 & 14.91 & 12.25 & 12.57 & 12.20 & 12.18 & 12.01 & 12.52 & 11.81 & 15.41 \\
\hline $\mathrm{K}_{2} \mathrm{O}$ & 0.55 & 0.46 & 0.22 & 0.51 & 0.32 & 0.47 & 0.30 & 0.57 & 0.29 & 0.58 & 0.16 \\
\hline Total (wt \%) & 97.16 & 96.91 & 97.08 & 96.81 & 97.71 & 97.57 & 98.82 & 96.96 & 97.17 & 97.39 & 96.95 \\
\hline \multicolumn{12}{|c|}{ Formulae based on 23 oxygen and $13 \mathrm{eCNK}$. } \\
\hline \multicolumn{12}{|l|}{ T site } \\
\hline $\mathrm{Si}$ & 6.77 & 6.87 & 6.84 & 6.84 & 6.84 & 6.86 & 6.66 & 6.79 & 6.90 & 6.86 & 7.07 \\
\hline $\mathrm{Ti}$ & 0.06 & 0.07 & 0.12 & 0.07 & 0.07 & 0.08 & 0.08 & 0.07 & 0.06 & 0.07 & 0.08 \\
\hline $\mathrm{Fe}^{3+}$ & 0.96 & 0.84 & 1.37 & 0.90 & 0.86 & 0.82 & 1.24 & 0.81 & 0.83 & 0.61 & 1.14 \\
\hline $\mathrm{Mg}$ & 2.59 & 2.75 & 3.16 & 2.68 & 2.72 & 2.66 & 2.60 & 2.64 & 2.72 & 2.59 & 3.26 \\
\hline $\mathrm{Fe}^{2+}$ & 1.13 & 1.01 & 0.24 & 1.05 & 1.07 & 1.15 & 0.83 & 1.19 & 1.08 & 1.39 & 0.34 \\
\hline $\mathrm{Mn}$ & 0.09 & 0.09 & 0.05 & 0.09 & 0.09 & 0.10 & 0.10 & 0.09 & 0.07 & 0.06 & 0.05 \\
\hline \multicolumn{12}{|l|}{ B site } \\
\hline $\mathrm{Ca}$ & 1.81 & 1.76 & 1.57 & 1.75 & 1.81 & 1.81 & 1.71 & 1.85 & 1.79 & 1.87 & 1.60 \\
\hline $\mathrm{Na}$ & 0.19 & 0.24 & 0.32 & 0.25 & 0.19 & 0.19 & 0.29 & 0.15 & 0.21 & 0.13 & 0.27 \\
\hline \multicolumn{12}{|l|}{ A site } \\
\hline $\mathrm{Na}$ & 0.06 & 0.06 & 0.00 & 0.06 & 0.11 & 0.07 & 0.03 & 0.10 & 0.07 & 0.14 & 0.00 \\
\hline K & 0.10 & 0.09 & 0.04 & 0.10 & 0.06 & 0.09 & 0.05 & 0.11 & 0.05 & 0.11 & 0.03 \\
\hline \multicolumn{12}{|c|}{ Pressure $(\mathrm{kbar})$, temperature $\left({ }^{\circ} \mathrm{C}\right)$, and depth $(\mathrm{km})$ of emplacement at a crustal density of $2.95 \mathrm{~g} / \mathrm{cm}^{3}$} \\
\hline $\mathrm{P}_{\text {EL98 }}$ & 4.6 & 6.3 & 4.7 & 6.0 & 4.8 & 4.9 & 5.6 & 4.4 & 5.8 & 5.3 & 5.1 \\
\hline $\mathrm{P}_{\mathrm{H} 87}$ & 3.1 & 3.0 & 2.1 & 3.0 & 2.8 & 2.8 & 3.6 & 3.2 & 2.8 & 3.3 & 1.2 \\
\hline $\mathbf{P}_{\text {AS95_O84 }}$ & 4.0 & 3.8 & 2.7 & 3.8 & 3.6 & 3.6 & 4.3 & 4.0 & 3.6 & 4.1 & 2.2 \\
\hline Depth & 13.4 & 12.9 & 9.0 & 12.9 & 12.4 & 12.1 & 14.6 & 13.4 & 12.3 & 13.8 & 7.4 \\
\hline Spot & TG51-4 & TG51-5 & TG51-6 & TG51-7 & TG51-8 & TG52-1 & TG52-2 & TG52-3 & TG52-4 & TG52-5 & TG52-6 \\
\hline $\mathrm{SiO}_{2}$ & 45.53 & 47.15 & 47.26 & 46.75 & 47.45 & 48.14 & 45.87 & 46.71 & 46.95 & 48.96 & 46.77 \\
\hline $\mathrm{TiO}_{2}$ & 0.64 & 0.72 & 0.71 & 0.57 & 0.79 & 0.98 & 0.84 & 0.83 & 0.68 & 1.00 & 0.64 \\
\hline $\mathrm{Al}_{2} \mathrm{O}_{3}$ & 8.18 & 8.06 & 8.02 & 8.02 & 8.30 & 6.99 & 8.61 & 8.38 & 8.04 & 6.70 & 8.06 \\
\hline $\mathrm{FeO}_{\text {total }}$ & 15.07 & 13.90 & 15.50 & 15.96 & 15.35 & 13.69 & 16.11 & 14.46 & 15.31 & 12.93 & 15.80 \\
\hline $\mathrm{MnO}$ & 0.55 & 0.52 & 0.59 & 0.53 & 0.60 & 0.47 & 0.72 & 0.53 & 0.76 & 0.40 & 0.74 \\
\hline $\mathrm{MgO}$ & 11.91 & 13.12 & 12.26 & 12.70 & 13.29 & 14.25 & 12.10 & 13.54 & 12.71 & 14.97 & 12.56 \\
\hline $\mathrm{CaO}$ & 11.49 & 11.36 & 11.37 & 11.27 & 10.93 & 10.87 & 11.44 & 10.92 & 11.57 & 10.54 & 11.51 \\
\hline $\mathrm{Na}_{2} \mathrm{O}$ & 1.03 & 1.33 & 0.96 & 0.94 & 0.97 & 0.94 & 1.06 & 1.30 & 1.07 & 1.05 & 1.06 \\
\hline $\mathrm{K}_{2} \mathrm{O}$ & 0.60 & 0.49 & 0.45 & 0.48 & 0.37 & 0.23 & 0.59 & 0.41 & 0.46 & 0.19 & 0.41 \\
\hline Total (wt \%) & 95.00 & 96.65 & 97.12 & 97.22 & 98.05 & 96.56 & 97.34 & 97.08 & 97.55 & 96.74 & 97.55 \\
\hline
\end{tabular}


Table 4. Cont.

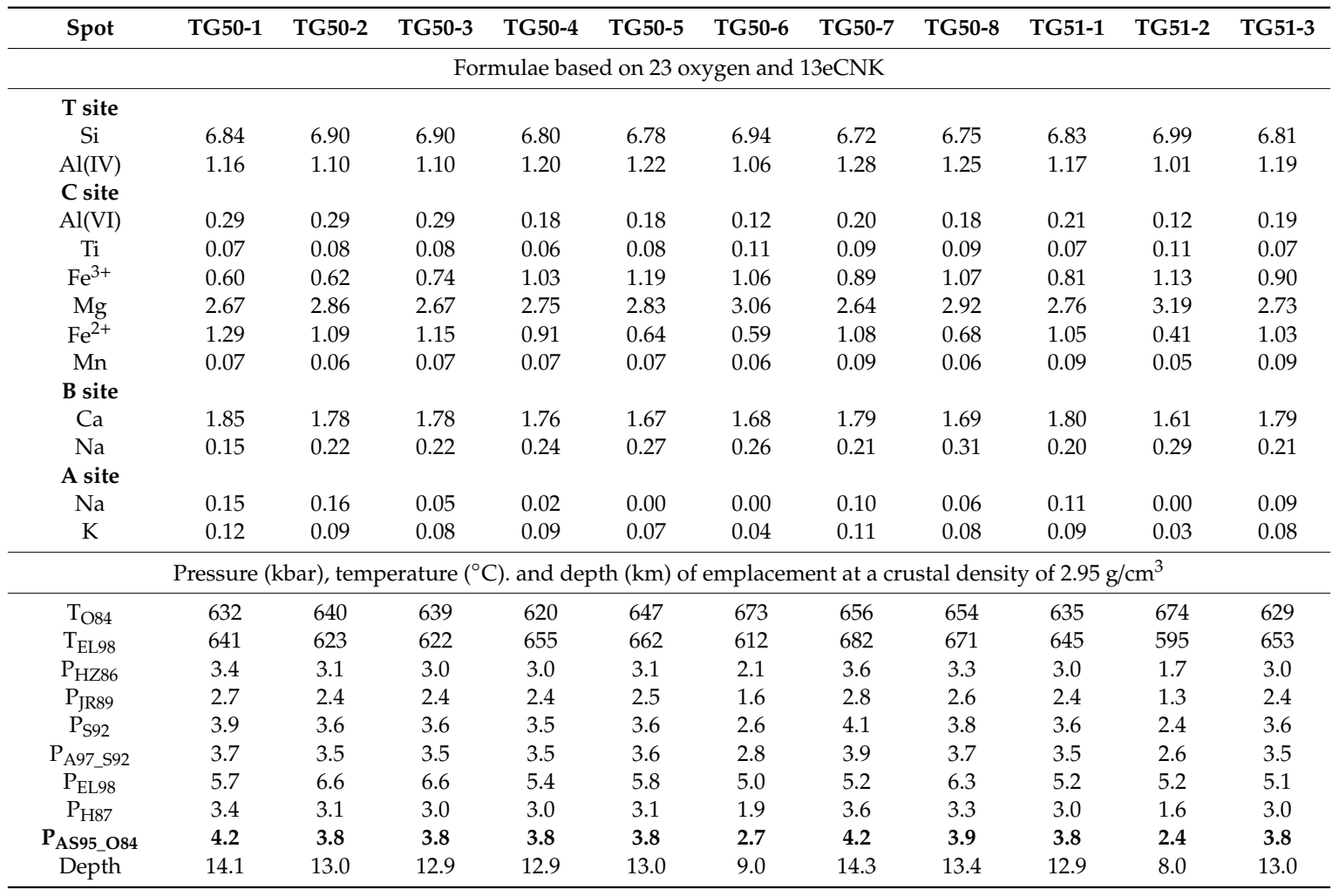

Note: Subscripts for T and P represent initials of names of authors and years of papers, see text for details. T, C, B, and A site represent the corresponding site in amphibole formula. The preferred values for pressure are marked in bold.

The structural formulae and stoichiometry of amphiboles were calculated based on an anhydrous basis assuming 23 oxygen and 13 cations, excluding $\mathrm{Ca}$, Na, and $\mathrm{K}(13 \mathrm{eCNK}) . \mathrm{Fe}^{3+}$ and $\mathrm{Fe}^{2+}$ contents were calculated assuming a charge of 46, as suggested by Leake et al. [56]. All of the Lameila amphiboles were calcic $\left(\mathrm{Na}_{\mathrm{B}}=0.13-0.32\right.$ atoms per formula unit $\left.(\mathrm{apfu}), \mathrm{Ca}_{\mathrm{B}}+\mathrm{Na}_{\mathrm{B}}=1.70-2.19 \mathrm{apfu}\right)$ and are classified as magnesiohornblende $\left(\mathrm{Mg} \#\left(\mathrm{Mg} /\left(\mathrm{Mg}+\mathrm{Fe}^{2+}\right)\right)=0.65-0.93, \mathrm{Si}=6.66-7.07 \mathrm{apfu}\right.$, Figure 8). Leake et al. [56] suggested that igneous amphiboles have a maximum of $\mathrm{Si}=7.3 \mathrm{apfu}$, and those with Si > 7.3 apfu were crystallized under sub-solidus conditions in the presence of a fluid or had been altered by hydrothermal fluids; therefore, the low Si signature of the Lameila hornblende suggests a magmatic origin, which is in accordance with the petrographic observations.

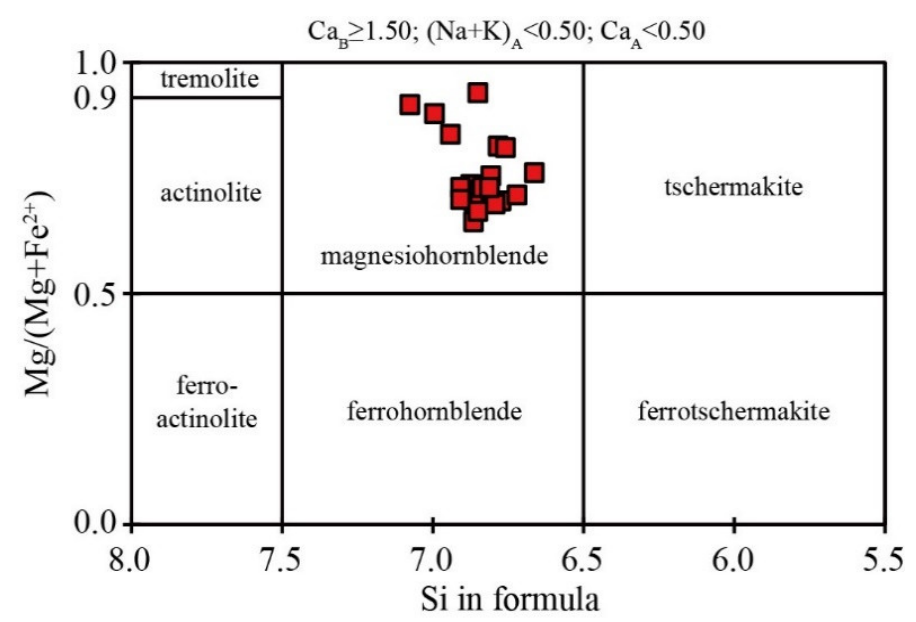

Figure 8. Classification of the Lameila amphiboles following Leake et al. [56]. 


\section{Discussion}

\subsection{Key Control Factors on the Variation of Amphibole Compositions}

The amphibole compositions are sensitive to physical-chemical conditions in magma systems, such as changing melt composition, pressure, temperature, volatile content, and co-crystallizing mineral phases [57]. Therefore, it is important to determine the dominating parameters that are responsible for the compositional variations of minerals. Variations in Al for the Lameila hornblendes are largely accommodated by the tetrahedral site $\left(\mathrm{Al}^{\mathrm{IV}}=0.93-1.34 \mathrm{apfu}\right)$. The amount of $\mathrm{Al}$ in the $\mathrm{C}$ site $\left(\mathrm{Al}^{\mathrm{VI}}=0.05-0.29 \mathrm{apfu}\right)$ is minor. Atom exchanges, such as the Al-Tschermak exchange, Ti-Tschermak exchange, the edenite exchange, and the plagioclase substitution are proposed to be significant mechanisms controlling compositional variations in amphiboles.

Elemental correlation diagrams of the Lameila amphiboles (Figure 9) imply that the contributions from both the pressure-sensitive Al-Tschermak exchange and the temperature-sensitive Ti-Tschermak exchange were negligible, as evidenced by the lack of covariations of $\mathrm{Al}^{\mathrm{VI}}$ and $\mathrm{Ti}_{C}$ with $\mathrm{Al}^{\mathrm{IV}}$ (Figure 9a,b). Instead, the slopes of the regression lines in the $(\mathrm{Na}+\mathrm{Ca})_{\mathrm{A}}-\mathrm{Al}^{\mathrm{IV}}$ and the $\mathrm{Ca}_{\mathrm{B}}-\mathrm{Al}^{\mathrm{IV}}$ diagrams (Figure $\left.9 \mathrm{~b}, \mathrm{c}\right)$ suggest that about $55 \%$ of the $\mathrm{Al}^{\mathrm{IV}}$ variations were accommodated by the temperature-sensitive edenite exchange and plagioclase substitution. The remaining proportions for the $\mathrm{Al}^{\mathrm{IV}}$ and total $\mathrm{Al}$ variation may have been controlled by some other factors, such as fractional crystallization of the host magma, or changes in the redox state during the crystallization of the Lameila amphiboles (Figure 9d-f). The effects of the fractional crystallization processes and the changes of the redox state on Al variation may have somewhat overlapped with those of the edenite exchange and plagioclase substitution; however, the extent to which the former two factors contributed to the Al variations of the Lameila amphiboles cannot be quantitatively evaluated in the current work. Nevertheless, the existence of the temperature-sensitive edenite exchange and the plagioclase substitution in the Lameila samples demands temperature correction for the Al-in-hornblende barometer.
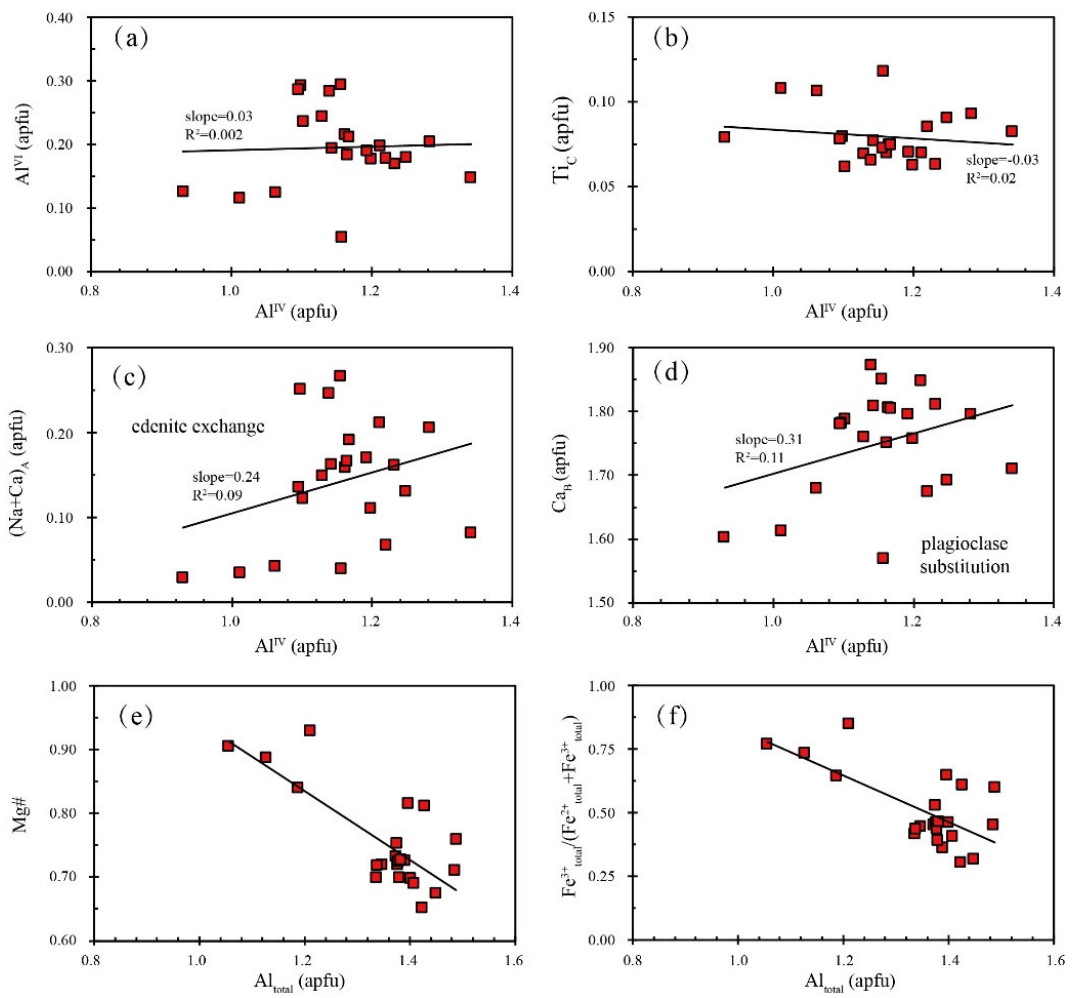

Figure 9. Composition variation diagrams for the Lameila amphiboles. (a) $\mathrm{Al}^{\mathrm{VI}}-\mathrm{Al}^{\mathrm{IV}}$ diagram; (b) $\mathrm{Ti}_{\mathrm{C}}-\mathrm{Al}^{\mathrm{IV}}$ diagram; (c) $(\mathrm{Na}+\mathrm{Ca})_{\mathrm{A}}-\mathrm{Al}^{\mathrm{IV}}$ diagram; (d) $\mathrm{Ca}_{\mathrm{B}}-\mathrm{Al}^{\mathrm{IV}}$ diagram; (e) $\mathrm{Mg} \#-\mathrm{Al}_{\text {total }}$ diagram; (f) $\mathrm{Fe}^{3+}{ }_{\text {total }} / \mathrm{Fe}^{2+}{ }_{\text {total }}-\mathrm{Al}_{\text {total }}$ diagram. 


\subsection{Al-in-Hornblende Barometer and Depth of the Lameila Pluton Emplacement}

Empirical calibration and experimental studies show that the total Al composition in calcic amphiboles increases linearly with crystallization pressure. This phenomenon forms the foundation of Al-based barometers for calcic amphiboles [57-61]. An important prerequisite for the applications of Al-in-hornblende barometers is an appropriate buffering assemblage (amphibole + plagioclase + K-feldspar/quartz, with medium to high oxygen fugacity). The lithology and petrography of the Lameila pluton (Figure 4) show that the above buffering assemblage was satisfied. In addition, the magma in which the Lameila amphiboles crystallized had a high oxygen fugacity. This inference is supported by the elevated $\mathrm{Ce} / \mathrm{Ce}^{*}$ and $\mathrm{Eu} / \mathrm{Eu}^{*}$ in the Lameila zircons (Figure 10) and the occurrence of primary magnetite grains within and along the margins of amphibole crystals (Figure 4c).

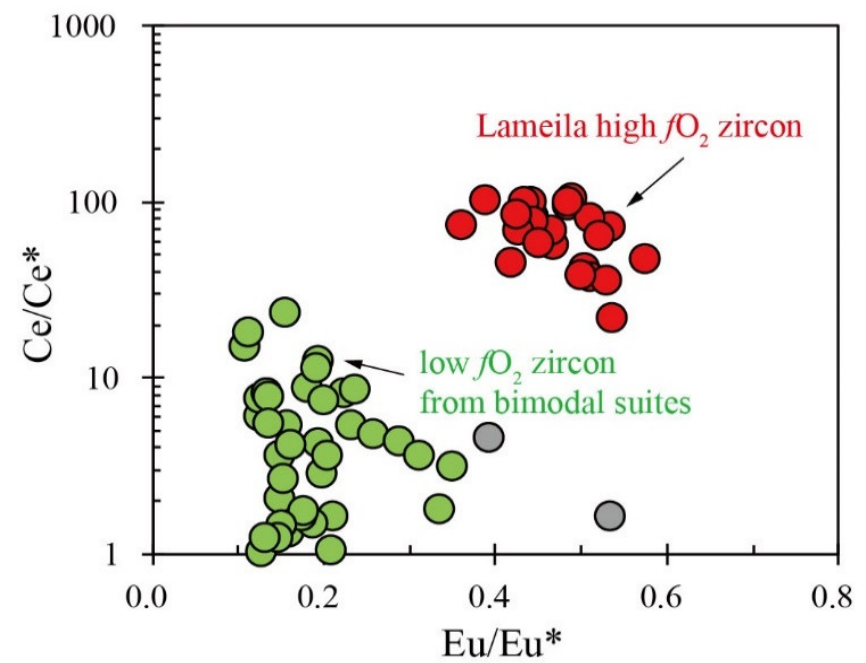

Figure 10. Plot of $\mathrm{Ce} / \mathrm{Ce}^{*}$ versus $\mathrm{Eu} / \mathrm{Eu}^{*}$ for zircons for the Lameila pluton. The data for bimodal volcanic suites are from Liu et al. [37].

We first calculated the emplacement pressure using Al-in-hornblende barometers without temperature correction. For this purpose, we employed several widely used barometers, including calibrations of $P_{\mathrm{HZ} 86}$ [61], $\mathrm{P}_{\mathrm{H} 87}$ [60], $\mathrm{P}_{\mathrm{JR} 89}$ [59], $\mathrm{P}_{\mathrm{S} 92}$ [62], and $\mathrm{P}_{\mathrm{EL} 98}$ [58]. Results of the calculated pressures are shown in Table 4.

We then used the temperature-corrected calibration of Anderson and Smith [57] to evaluate the emplacement pressure. The temperatures for amphibole crystallization were estimated by employing several kinds of methods (Figure 11). The first $\left(\mathrm{T}_{\mathrm{O} 84}\right)$ was the Ti-in-amphibole calibration of Otten [63], which was first reported by Helz [64]. The second $\left(\mathrm{T}_{\mathrm{EL} 98}\right)$ was the amphibole thermometer of Ernst and Liu [58]. Results of these two thermometers were further compared with temperatures $\left(\mathrm{T}_{\text {Ti-in-zircon }}\right)$ derived from the Ti-in-zircon thermometer [65]. Ranges of $\mathrm{T}_{\mathrm{O} 84}\left(619-687^{\circ} \mathrm{C}\right.$, average $\left.=642{ }^{\circ} \mathrm{C}\right)$ and $\mathrm{T}_{\mathrm{EL} 98}\left(569-701{ }^{\circ} \mathrm{C}\right.$, average $\left.=641^{\circ} \mathrm{C}\right)$ largely overlapped with each other, but the results of $\mathrm{T}_{\text {EL98 }}$ were more scattered than $\mathrm{T}_{\mathrm{O} 84}$. The $\mathrm{T}_{\mathrm{Ti} \text {-in-zircon }}$ can record the temperature at which a zircon grain crystallizes in the host magma. Stages for zircon crystallization in an intermediate/felsic magma could be either early or late, and thus $\mathrm{T}_{\mathrm{Ti} \text {-in-zircon }}$ of different zircon grains could record whole-stage temperatures of a magma system. Amphiboles crystallized early in dioritic magma during emplacement, and overlap between the ranges of $\mathrm{T}_{\mathrm{Ti} \text {-in-zircon }}$ and $\mathrm{T}_{\mathrm{O} 84}$ was the best estimation for the temperatures for amphibole crystallization. The trend of $\mathrm{T}_{\text {Ti-in-zircon }}$ with increasingly younger ages was likely the result of heating by magma replenishment after the crystallization of zircon grains with older ages (Figure 11c). This batch of magma replenishment was recorded in the dark-colored amphibole-rich xenoliths of diorites that were in sharp contact with the host Lameila quartz diorites. The replenished magma probably delivered heat but not materials to the quartz diorites, which is evidenced by the linearly decreasing $\mathrm{Mg}$ with $\mathrm{Al}$ (Figure 9e). On this basis, the $\mathrm{T}_{\mathrm{O} 84}$ results are used in this study 
for correction of the Al-in-hornblende barometer $\left(\mathrm{P}_{\mathrm{AS95}} \mathrm{TO} 44\right)$ proposed by Anderson and Smith [57]. The corrected $\mathrm{P}_{\mathrm{AS95} \text { _TO84 }}$ ranged from 3.6 to $4.3 \mathrm{kbar}$ except for four outliers (2.2-2.7 kbar, Figure 11d). The outliers with elevated temperatures were likely the result of heating as recorded by the $\mathrm{T}_{\text {Ti-in-zircon }}$ trend (Figure 11c). The average value of the majority was $3.89 \pm 0.28 \mathrm{kbar}(\mathrm{n}=18, \mathrm{MSWD}=0.11)$, which was interpreted as the pressure of the Lameila pluton emplacement.
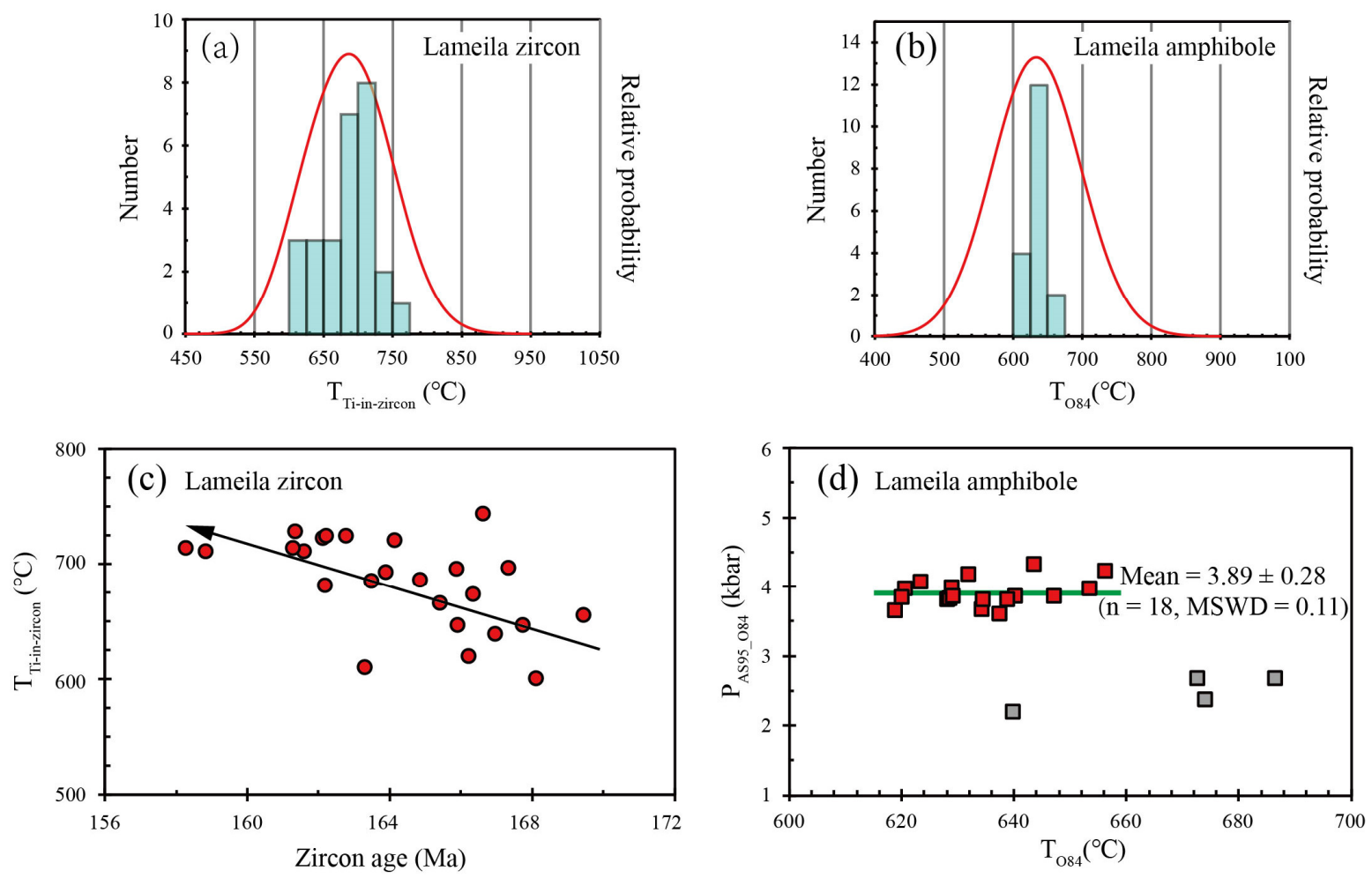

Figure 11. Plots of $\mathrm{T}_{\text {Ti-in-zircon }}(\mathbf{a}), \mathrm{T}_{\mathrm{O} 84}(\mathbf{b}), \mathrm{T}_{\mathrm{Ti} \text {-in-zircon }}$ versus zircon age (c), and $\mathrm{P}_{\mathrm{AS} 95 \text { _O84 }}$ versus $\mathrm{T}_{\mathrm{O} 84}$ (d) for the Lameila pluton. Please see text for explanations. In panel d, data used for the calculation of the mean value are shown as red squares, and the outliers are shown as grey squares.

Uncertainties for the pressure estimation were the followings: (1) analytical uncertainties are better than 1\%; (2) uncertainties with the Al-in-hornblende calibration are within $0.6 \mathrm{kbar}$; (3) the standard deviation for $\mathrm{P}_{\text {AS95_TO84 }}$ results is 0.19 kbar. Taken all together, the uncertainties for the pressure would be better than $0.83 \mathrm{kbar}$. As a consequence, the pressure for the Lameila pluton is $3.89 \pm 0.83 \mathrm{kbar}$. The average continental crust density is $2.700-2.875 \mathrm{~g} / \mathrm{cm}^{3}$, and the oceanic crust is $2.700-2.950 \mathrm{~g} / \mathrm{cm}^{3}$ [66]. The density of the oceanic arc crust in the BNO is not clear, but should be between the above two crusts. If we employ a conservative density of $2.950 \mathrm{~g} / \mathrm{cm}^{3}$, the depth of the Lameila pluton emplacement would be $13.2 \pm 2.8 \mathrm{~km}$.

\subsection{Mechanism for the Crustal Thickening of the Jurassic Oceanic Arcin the BNO}

Previous studies on high-Mg volcanic rocks within the BNS suggest a Jurassic oceanic arc that formed during the intra-oceanic subduction of the BNO. However, the configuration of this oceanic arc, including the crustal thickness, is not clear. As mentioned in Sections 5.1 and 5.2, this oceanic arc in the BNO has grown significantly. The Al-in-hornblende barometer shows that the $164 \mathrm{Ma}$ Lameila pluton in western Tibet intrudes in the upper plate of the Jurassic oceanic subduction system within the $\mathrm{BNO}$ at a depth of about $13 \mathrm{~km}$. This emplacement depth is the minimum crustal thickness of the Jurassic oceanic arc within the $\mathrm{BNO}$, since the thickness of the crust underneath the emplacement level of the Lameila pluton is unknown. This depth of emplacement implies that the Jurassic oceanic arc crust has thickened significantly since the initial development of this oceanic arc on the oceanic crust of 
the BNO. According to studies on both modern and paleo-oceanic arcs around the world, arc crust thicknesses depend on the thickness of pre-arc basement, tectonic extension or shortening, and arc maturity (magmatic addition and evolution) [67]. The pre-arc basement crust consists of mainly mafic oceanic crust and minor sedimentary cover. Studies on the ophiolites show that the composition of the oceanic crust is dominated by regular N-MORB and E-MORB type mafic rocks, and that oceanic crust related to OIB (oceanic island basalt) or oceanic plateaus are not recognized in the Shiquanhe area $[10,68]$. Therefore, the thickness of pre-arc oceanic crust is most likely comparable to that of the average oceanic crust $(6.5 \mathrm{~km})$ [69]. Rocks with levels higher than the Lameila pluton have been removed, thus much of the Lameila pluton is exposed at the surface (Figure 4a), and the outcrops of sedimentary rocks with pre-arc ages are extremely limited in the vicinity of the Lameila pluton and the Shiquanhe high-Mg andesite (Figure 3). Therefore, the pre-arc sedimentary covers on the floor of the BNO may be quite thin and thus their contribution to pre-arc crustal thickness is negligible. The effect of tectonic shortening on the thickening of the Jurassic oceanic arc in the BNO could not be quantitatively evaluated in this work due to the very limited preservation of structural traces.

Alternatively, magmatism may play a considerable role in the thickening of the Jurassic oceanic arc within the BNO. Studies on modern oceanic arcs, such as the Izu-Bonin-Marina [70-73], the Lesser Antilles [74-76], the Tonga-Kermadec [77-79], and the Aleutian [80-82] arcs show a variation of crustal thickness $(9-35 \mathrm{~km})$ within a single arc or between different arcs. In addition, an important finding of these studies is that the thick part of an arc is always related to high magmatic flux. This phenomenon implies that magmatic addition is a dominant mechanism responsible for oceanic arc crustal thickening. The lithological assemblage of the Jurassic oceanic arc in the BNS comprises only magmatic rocks, such as the Lameila quartz diorites, the Shiquanhe and Daruco high-Mg andesites, and the Dingqing high-Mg quartz gabbro/diabase. Pending additional studies of detailed tectonic evolution, we tentatively propose the crustal thickening of the Jurassic oceanic arc in the BNO to be a result of magmatic addition.

\subsection{Oceanic Arc Development and Two Contrasting Sources for Magmatism}

The east-west trending BNS in the Tibetan Plateau is a belt more than $1200 \mathrm{~km}$ long consisting of scattered ophiolitic fragments that are associated with thick sequences of Jurassic flysch. The BNS is extraordinarily wide, especially near Amdo in eastern Tibet and between Rutog and Shiquanhe in western Tibet (Figure 1). Besides the ophiolitic fragments and the Jurassic flysch, there are also micro-continental blocks such as the Amdo micro-block, and possibly oceanic arc blocks within the BNS. The mechanism incorporating these micro-blocks into the BNS is unclear.

In contrast to the well-exposed Amdo micro-block, surface traces of the oceanic arc rocks in the BNS are highly obscure. Previous recognition of BNS oceanic arc rocks has been mainly based on geochemical evidence from the volcanic rocks that are associated with ophiolitic fragments (SSZ type ophiolite), and suggests that an oceanic subduction occurred within the BNO [10,31,83-85]. Importantly, the discoveries of boninitic rocks near Dingqing [17], Daruco [20], Shiquanhe [19], and Rutog [18] in the BNS provide further evidence of the existence of an oceanic arc within the BNO. However, the extremely localized outcrops of these volcanic rocks make it hard to trace them in the field, and thus hamper further studies on the development and configuration of this arc. The new data from the Lameila pluton of this study have three implications for the tectonic evolution of the BNO. Firstly, this study recognizes the intrusive part of the oceanic arc, and in combination with the previously reported extrusive rocks, further confirms the existence of a Jurassic oceanic arc within the BNO. Secondly, the emplacement depth of the Lameila pluton suggests that the crust of the oceanic arc within the BNO was significantly thickened during the end of the middle Jurassic. Thirdly, the zircon Lu-Hf isotopes of the Lameila pluton, together with that of the previously reported high-Mg andesites within the BNS, imply diverse sources for the oceanic arc magmatism as shown below.

Studies on the high-Mg andesites from Shiquanhe (Figure 3) and Daruco (Figure 1) show arc-type geochemical compositions and enriched $\mathrm{Nd}\left(\varepsilon_{\mathrm{Nd}}(\mathrm{t})=-8.7\right.$ to -13.4$)$ and $\mathrm{Hf}\left(\varepsilon_{\mathrm{Hf}}(\mathrm{t})=-7.4\right.$ to -16.2$)$ 
isotopes. It has been proposed that these high-Mg andesites are formed by the melting of subducted sediments (Figure 12) and subsequent interaction with overlying mantle peridotites during subduction initiation $[19,20]$. In contrast with the enriched isotopes of the above high-Mg andesites, zircon Lu-Hf isotopes of the Lameila pluton were depleted, with $\varepsilon_{\mathrm{Hf}}(\mathrm{t})$ ranging from 10.5 to 13.9 . The depleted $\mathrm{Lu}-\mathrm{Hf}$ isotopes of the Lameila pluton clearly indicate that they were formed by the partial melting of a depleted-mantle source, most likely the depleted-mantle wedge peridotites above the oceanic subduction zone in the BNO (Figure 12). Synchronous with this oceanic arc in the BNO, a Jurassic continental arc (Figure 1) in the south margin of the Qiangtang block has also developed, as suggested by many previous researchers $[13,15,16,29,38,39]$. These two arcs suggest the occurrence of two subduction zones during the Middle Jurassic, namely, an oceanic-continental subduction zone between the BNO and the Qiangtang block in the north, and an oceanic subduction zone within the BNO in the south (Figure 12). The Jurassic oceanic arc presented in this study and the Amdo micro-continental block (Figure 1) were accreted onto the southern margin of the Qiangtang block during the continued subduction of the BNO, or during the Lhasa-Qiangtang collision. The accretion of the oceanic arc and micro-block could lead to a wide Bangong-Nujiang suture in central Tibet (Figure 2d).

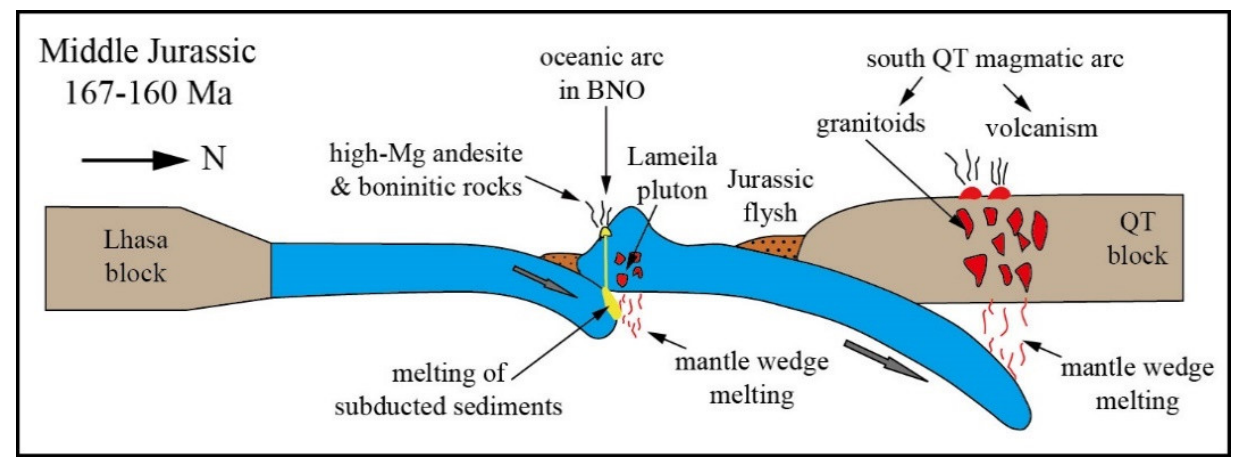

Figure 12. Tectonic model showing the Middle Jurassic Bangong-Nujiang Ocean (BNO) subduction, including oceanic subduction within the BNO and the subduction of the BNO beneath the south margin of the Qiangtang (QT) block. Associated with these two subduction zones are two arcs, one oceanic arc developed within the BNO and the other formed in the south margin of the Qiangtang block.

\section{Conclusions}

This work reports a study of the plutonic part of the Jurassic oceanic arc within the Bangong-Nujiang suture in western Tibet. The Lameila pluton consists mainly of quartz diorites with a zircon $\mathrm{U}-\mathrm{Pb}$ age of $164 \mathrm{Ma}$. In addition, the zircon $\varepsilon_{\mathrm{Hf}}(\mathrm{t})$ values of 10.5-13.9 indicate that the Lameila pluton was most likely sourced from the depleted-mantle wedge. Temperature-corrected Al-in-hornblende pressure was $3.9 \pm 0.8 \mathrm{kbar}$, and the corresponding depth of emplacement was $13 \pm 3 \mathrm{~km}$. The thickness of the Jurassic oceanic arc crust must have doubled since the initial growth of the oceanic arc on the Bangong-Nujiang Ocean crust, with a thickness of $6.5 \mathrm{~km}$ during the Middle Jurassic.

Author Contributions: Conceptualization, D.-L.L. and S.-Y.J.; Field work in the Tibetan Plateau, D.-L.L. and M.S.; Data Curation, D.-L.L.; Writing-original draft, D.-L.L., S.-Y.J.; Writing-review \& editing, S.-Y.J.; Funding Acquisition, D.-L.L. and S.-Y.J.

Funding: This work was financially supported by the National Natural Science Foundation of China (41702212), the Fundamental Research Funds for the National University, China University of Geosciences (Wuhan) (CUGL170816 and CUGQYZX1745), and the special fund from the State Key Laboratory of Geological Processes and Mineral Resources, China University of Geosciences (No. MSFGPMR03-2).

Acknowledgments: We thank Zheng-Han Li for his assistance in the field, and Kui-Dong Zhao and Shui-Yuan Yang for their assistance with $\mathrm{U}-\mathrm{Pb}-\mathrm{Lu}-\mathrm{Hf}$ isotopes and mineral chemistry measurements. We thank the editors for editorial handling and suggestions. We thank three anonymous reviewers for useful comments and suggestions. 
Conflicts of Interest: The authors declare no conflict of interest. The funders had no role in the design of the study; in the collection, analyses, or interpretation of data; in the writing of the manuscript, or in the decision to publish the results.

\section{References}

1. Fielding, E.; Isacks, B.; Barazangi, M.; Duncan, C. How flat is Tibet? Geology 1994, 22, 163-167. [CrossRef]

2. Zhang, Z.; Deng, Y.; Teng, J.; Wang, C.; Gao, R.; Chen, Y.; Fan, W. An overview of the crustal structure of the Tibetan plateau after 35 years of deep seismic soundings. J. Asian Earth Sci. 2011, 40, 977-989. [CrossRef]

3. Kapp, P.; DeCelles, P.G. Mesozoic-Cenozoic geological evolution of the Himalayan-Tibetan orogen and working tectonic hypotheses. Am. J. Sci. 2019, 319, 159-254. [CrossRef]

4. Zhu, D.-C.; Zhao, Z.-D.; Niu, Y.; Dilek, Y.; Hou, Z.-Q.; Mo, X.-X. The origin and pre-Cenozoic evolution of the Tibetan Plateau. Gondwana Res. 2013, 23, 1429-1454. [CrossRef]

5. Yin, A.; Harrison, T.M. Geologic evolution of the Himalayan-Tibetan Orogen. Annu. Rev. Earth Plant Sci. 2000, 28, 211-280. [CrossRef]

6. Li, S.; Yin, C.; Guilmette, C.; Ding, L.; Zhang, J. Birth and demise of the Bangong-Nujiang Tethyan Ocean: A review from the Gerze area of central Tibet. Earth Sci. Rev. 2019, 198, 102907. [CrossRef]

7. Zhu, D.-C.; Li, S.-M.; Cawood, P.A.; Wang, Q.; Zhao, Z.-D.; Liu, S.-A.; Wang, L.-Q. Assembly of the Lhasa and Qiangtang terranes in central Tibet by divergent double subduction. Lithos 2016, 245, 7-17. [CrossRef]

8. Kapp, P.; Murphy, M.A.; Yin, A.; Harrison, T.M.; Ding, L.; Guo, J.H. Mesozoic and Cenozoic tectonic evolution of the Shiquanhe area of western Tibet. Tectonics 2003, 22, 1-24. [CrossRef]

9. Fan, J.-J.; Li, C.; Liu, J.-H.; Wang, M.; Liu, Y.-M.; Xie, C.-M. The Middle Triassic evolution of the Bangong-Nujiang Tethyan Ocean: Evidence from analyses of OIB-type basalts and OIB-derived phonolites in northern Tibet. Int. J. Earth Sci. 2018, 107, 1755-1775. [CrossRef]

10. Wang, B.-D.; Wang, L.-Q.; Chung, S.-L.; Chen, J.-L.; Yin, F.-G.; Liu, H.; Li, X.-B.; Chen, L.-K. Evolution of the Bangong-Nujiang Tethyan ocean: Insights from the geochronology and geochemistry of mafic rocks within ophiolites. Lithos 2016, 245, 18-33. [CrossRef]

11. Zhang, K.-J.; Xia, B.; Zhang, Y.-X.; Liu, W.-L.; Zeng, L.; Li, J.-F.; Xu, L.-F. Central Tibetan Meso-Tethyan oceanic plateau. Lithos 2014, 210-211, 278-288. [CrossRef]

12. Tian, X.; Wu, Q.; Zhang, Z.; Teng, J.; Zeng, R. Joint imaging by teleseismic converted and multiple waves and its application in the INDEPTH-III passive seismic array. Geophys. Res. Lett. 2005, 32. [CrossRef]

13. Li, X.-K.; Chen, J.; Wang, R.-C.; Li, C. Temporal and spatial variations of Late Mesozoic granitoids in the SW Qiangtang, Tibet: Implications for crustal architecture, Meso-Tethyan evolution and regional mineralization. Earth Sci. Rev. 2018, 185, 374-396. [CrossRef]

14. Hao, L.-L.; Wang, Q.; Zhang, C.; Ou, Q.; Yang, J.-H.; Dan, W.; Jiang, Z.-Q. Oceanic plateau subduction during closure of the Bangong-Nujiang Tethyan Ocean: Insights from central Tibetan volcanic rocks. GSA Bull. 2018, 131, 864-880. [CrossRef]

15. Liu, D.; Shi, R.; Ding, L.; Huang, Q.; Zhang, X.; Yue, Y.; Zhang, L. Zircon U-Pb age and Hf isotopic compositions of Mesozoic granitoids in southern Qiangtang, Tibet: Implications for the subduction of the Bangong-Nujiang Tethyan Ocean. Gondwana Res. 2017, 41, 157-172. [CrossRef]

16. Li, J.-X.; Qin, K.-Z.; Li, G.-M.; Richards, J.P.; Zhao, J.-X.; Cao, M.-J. Geochronology, geochemistry, and zircon Hf isotopic compositions of Mesozoic intermediate-felsic intrusions in central Tibet: Petrogenetic and tectonic implications. Lithos 2014, 198-199, 77-91. [CrossRef]

17. Zhang, Q.; Yang, R.-Y. The geochemical characteristics of intrusion of boninitic series from Dingqing, Xizang. Acta Petrol. Sin. 1987, 2, 64-74.

18. Shi, R.D.; Yang, J.S.; Xu, Z.Q.; Qi, X.X. Discovery of the boninite series volcanic rocks in the Bangong Lake ophiolite mélange, western Tibet, and its tectonic implications. Chin. Sci. Bull. 2004, 49, 1272-1278. [CrossRef]

19. Liu, W.-L.; Huang, Q.-T.; Gu, M.; Zhong, Y.; Zhou, R.; Gu, X.-D.; Zheng, H.; Liu, J.-N.; Lu, X.-X.; Xia, B. Origin and tectonic implications of the Shiquanhe high-Mg andesite, western Bangong suture, Tibet. Gondwana Res. 2018, 60, 1-14. [CrossRef] 
20. Zeng, Y.-C.; Chen, J.-L.; Xu, J.-F.; Wang, B.-D.; Huang, F. Sediment melting during subduction initiation: Geochronological and geochemical evidence from the Darutso high-Mg andesites within ophiolite melange, central Tibet. Geochem. Geophys. Geosys. 2016, 17, 4859-4877. [CrossRef]

21. Pan, G.; Wang, L.; Li, R.; Yuan, S.; Ji, W.; Yin, F.; Zhang, W.; Wang, B. Tectonic evolution of the Qinghai-Tibet plateau. J. Asian Earth Sci. 2012, 53, 3-14. [CrossRef]

22. Pan, G.T.; Ding, J.; Yao, D.S. Geological Map (1:1500,000) of Qinghai-Xizang (Tibet) Plateau and Adjacent Areas; Chengdu Cartographic Publishing House: Chengdu, China, 2004.

23. Zhang, Y.-X.; Li, Z.-W.; Zhu, L.-D.; Zhang, K.-J.; Yang, W.-G.; Jin, X. Newly discovered eclogites from the Bangong Meso-Tethyan suture zone (Gaize, central Tibet, western China): Mineralogy, geochemistry, geochronology, and tectonic implications. Int. Geol. Rev. 2015, 1-14. [CrossRef]

24. Zhong, Y.; Liu, W.-L.; Xia, B.; Liu, J.-N.; Guan, Y.; Yin, Z.-X.; Huang, Q.-T. Geochemistry and geochronology of the Mesozoic Lanong ophiolitic mélange, northern Tibet: Implications for petrogenesis and tectonic evolution. Lithos 2017, 292, 111-131. [CrossRef]

25. Zhou, Y.; Cheng, X.; Yu, L.; Yang, X.; Su, H.; Peng, X.; Xue, Y.; Li, Y.; Ye, Y.; Zhang, J.; et al. Paleomagnetic study on the Triassic rocks from the Lhasa Terrane, Tibet, and its paleogeographic implications. J. Asian Earth Sci. 2016, 121, 108-119. [CrossRef]

26. Cao, Y.; Sun, Z.; Li, H.; Pei, J.; Liu, D.; Zhang, L.; Ye, X.; Zheng, Y.; He, X.; Ge, C.; et al. New paleomagnetic results from middle Jurassic limestones of the Qiangtang Terrane, Tibet: Constraints on the evolution of the Bangong-Nujiang Ocean. Tectonics 2019, 38, 215-232. [CrossRef]

27. Song, P.; Ding, L.; Li, Z.; Lippert, P.C.; Yang, T.; Zhao, X.; Fu, J.; Yue, Y. Late Triassic paleolatitude of the Qiangtang block: Implications for the closure of the Paleo-Tethys Ocean. Earth Planet Sci. Lett. 2015, 424, 69-83. [CrossRef]

28. Li, J.-X.; Qin, K.-Z.; Li, G.-M.; Xiao, B.; Zhao, J.-X.; Cao, M.-J.; Chen, L. Petrogenesis of ore-bearing porphyries from the Duolong porphyry $\mathrm{Cu}-\mathrm{Au}$ deposit, central Tibet: Evidence from $\mathrm{U}-\mathrm{Pb}$ geochronology, petrochemistry and Sr-Nd-Hf-O isotope characteristics. Lithos 2013, 160-161, 216-227. [CrossRef]

29. Zhang, K.-J.; Zhang, Y.-X.; Tang, X.-C.; Xia, B. Late Mesozoic tectonic evolution and growth of the Tibetan plateau prior to the Indo-Asian collision. Earth Sci. Rev. 2012, 114, 236-249. [CrossRef]

30. Zhou, M.F.; Malpas, J.; Robinson, P.T.; Reynolds, P.H. The dynamothermal aureole of the Donqiao ophiolite (northern Tibet). Can. J. Earth Sci. 1997, 34, 59-65. [CrossRef]

31. Shi, R.D. SHRIMP dating of the Bangong Lake SSZ-type ophiolite: Constraints on the closure time of ocean in the Bangong Lake-Nujiang River, northwestern Tibet. Chin. Sci. Bull. 2007, 52, 936-941. [CrossRef]

32. Shi, R.D. Recognition of the Bangong Lake MOR-and SSZ-Type Ophiolites in the Northwestern Tibet Plateau and Its Tectonic Significance. Ph.D. Thesis, Institute of Geology, Chinese Academy of Geological Sciences, Beijing, China, 2005.

33. Zhu, D.C.; Zhao, Z.D.; Niu, Y.L.; Mo, X.X.; Chung, S.L.; Hou, Z.Q.; Wang, L.Q.; Wu, F.Y. The Lhasa Terrane: Record of a microcontinent and its histories of drift and growth. Earth Planet Sci. Lett. 2011, 301, 241-255. [CrossRef]

34. Girardeau, J.; Marcoux, J.; Allegre, C.J.; Bassoullet, J.P.; Youking, T.; Xuchang, X.; Yougong, Z.; Xibin, W. Tectonic environment and geodynamic significance of the Neo-Cimmerian Donqiao ophiolite, Bangong-Nujiang suture zone, Tibet. Nature 1984, 307, 27-31. [CrossRef]

35. Matte, P.; Tapponnier, P.; Arnaud, N.; Bourjot, L.; Avouac, J.P.; Vidal, P.; Liu, Q.; Pan, Y.S.; Wang, Y. Tectonics of western Tibet, between the Tarim and the Indus. Earth Planet Sci. Lett. 1996, 142, 311-330. [CrossRef]

36. Kapp, P.; DeCelles, P.G.; Gehrels, G.E.; Heizler, M.; Ding, L. Geological records of the Lhasa-Qiangtang and Indo-Asian collisions in the Nima area of central Tibet. Geol. Soc. Am. Bull. 2007, 119, 917-933. [CrossRef]

37. Liu, D.-L.; Shi, R.-D.; Ding, L.; Zou, H.-B. Late Cretaceous transition from subduction to collision along the Bangong-Nujiang Tethys: New volcanic constraints from central Tibet. Lithos 2018, 296, 452-470. [CrossRef]

38. Li, S.-M.; Zhu, D.-C.; Wang, Q.; Zhao, Z.-D.; Sui, Q.-L.; Liu, S.-A.; Liu, D.; Mo, X.-X. Northward subduction of Bangong-Nujiang Tethys: Insight from Late Jurassic intrusive rocks from Bangong Tso in western Tibet. Lithos 2014, 205, 284-297. [CrossRef]

39. Liu, D.; Huang, Q.; Fan, S.; Zhang, L.; Shi, R.; Ding, L. Subduction of the Bangong-Nujiang Ocean: Constraints from granites in the Bangong Co area, Tibet. Geol. J. 2014, 49, 188-206. [CrossRef]

40. Ratschbacher, L.; Frisch, W.; Liu, G.H.; Chen, C.S. Distributed Deformation in Southern and Western Tibet during and after the India-Asia Collision. J. Geophys. Res. Solid Earth 1994, 99, 19917-19945. [CrossRef] 
41. Qin, Y.; Li, D.; Liu, D.; Li, H. Opening Time of Middle Tethys Oceanic Basin: Constrained from Zircon U-Pb Dating of MOR-type Gabbro in Bangong Lake Ophiolite. Geotect. Met. 2017, 41, 1148-1157.

42. Zheng, Y.Y.; Xu, R.K.; Ma, G.T.; Gao, S.; Zhang, G.; Ma, X.; Ci, Q. Ages of generation and subduction of Shiquan River ophiolite: Restriction from SHRIMP zircon dating. Acta Petrol. Sin. 2006, 22, 895-904.

43. Kapp, P.; Yin, A.; Harrison, T.M.; Ding, L. Cretaceous-Tertiary shortening, basin development, and volcanism in central Tibet. Geol. Soc. Am. Bull. 2005, 117, 865-878. [CrossRef]

44. Liu, W.-L.; Xia, B.; Zhong, Y.; Cai, J.-X.; Li, J.-F.; Liu, H.-F.; Cai, Z.-R.; Sun, Z.-L. Age and composition of the Rebang Co and Julu ophiolites, central Tibet: Implications for the evolution of the Bangong Meso-Tethys. Int. Geol. Rev. 2014, 56, 430-447. [CrossRef]

45. Wiedenbeck, M.; Alle, P.; Corfu, F.; Griffin, W.; Meier, M.; Oberli, F.; Quadt, A.v.; Roddick, J.; Spiegel, W. Three natural zircon standards for U-Th-Pb, Lu-Hf, trace element and REE analyses. Geostand. Newsl. 1995, 19, 1-23. [CrossRef]

46. Liu, Y.; Gao, S.; Hu, Z.; Gao, C.; Zong, K.; Wang, D. Continental and Oceanic Crust Recycling-induced Melt-Peridotite Interactions in the Trans-North China Orogen: U-Pb Dating, Hf Isotopes and Trace Elements in Zircons from Mantle Xenoliths. J. Petrol. 2010, 51, 537-571. [CrossRef]

47. Ludwig, K.R. User's Manual for Isoplot 3.00: A Geochronological Toolkit for Microsoft Excel; Berkeley Geochronology Center Special Publication: Berkeley, CA, USA, 2003.

48. Chu, N.-C.; Taylor, R.N.; Chavagnac, V.; Nesbitt, R.W.; Boella, R.M.; Milton, J.A.; German, C.R.; Bayon, G.; Burton, K. Hf isotope ratio analysis using multi-collector inductively coupled plasma mass spectrometry: An evaluation of isobaric interference corrections. J. Anal. At. Spectrom. 2002, 17, 1567-1574. [CrossRef]

49. Li, X.; Tang, G.; Gong, B.; Yang, Y.; Hou, K.; Hu, Z.; Li, Q.; Liu, Y.; Li, W. Qinghu zircon: A working reference for microbeam analysis of $\mathrm{U}-\mathrm{Pb}$ age and $\mathrm{Hf}$ and $\mathrm{O}$ isotopes. Chin. Sci. Bull. 2013, 58, 4647-4654. [CrossRef]

50. Söderlund, U.; Patchett, P.J.; Vervoort, J.D.; Isachsen, C.E. The 176Lu decay constant determined by Lu-Hf and $\mathrm{U}-\mathrm{Pb}$ isotope systematics of Precambrian mafic intrusions. Earth Planet Sci. Lett. 2004, 219, 311-324. [CrossRef]

51. Bouvier, A.; Vervoort, J.D.; Patchett, P.J. The Lu-Hf and Sm-Nd isotopic composition of CHUR: Constraints from unequilibrated chondrites and implications for the bulk composition of terrestrial planets. Earth Planet Sci. Lett. 2008, 273, 48-57. [CrossRef]

52. Griffin, W.L.; Pearson, N.J.; Belousova, E.; Jackson, S.E.; van Achterbergh, E.; O'Reilly, S.Y.; Shee, S.R. The Hf isotope composition of cratonic mantle: LAM-MC-ICPMS analysis of zircon megacrysts in kimberlites. Geochim. Cosmochim. Acta 2000, 64, 133-147. [CrossRef]

53. Griffin, W.L.; Wang, X.; Jackson, S.E.; Pearson, N.J.; O’Reilly, S.Y.; Xu, X.; Zhou, X. Zircon chemistry and magma mixing, SE China: In-situ analysis of Hf isotopes, Tonglu and Pingtan igneous complexes. Lithos 2002, 61, 237-269. [CrossRef]

54. Zhang, R.-X.; Yang, S.-Y. A Mathematical Model for Determining Carbon Coating Thickness and Its Application in Electron Probe Microanalysis. Microsc. Microanal. 2016, 22, 1374-1380. [CrossRef] [PubMed]

55. Boynton, W.V. Cosmochemistry of the Rare Earth Elements: Meteorite Studies; Elsevier Science Publishing Company: Amsterdam, The Netherlands, 1984; pp. 63-114.

56. Leake, B.E.; Woolley, A.R.; Arps, C.E.S.; Birch, W.D.; Gilbert, M.C.; Grice, J.D.; Hawthorne, F.C.; Kato, A.; Kisch, H.J.; Krivovichev, V.G.; et al. Nomenclature of amphiboles; report of the subcommittee on amphiboles of the International Mineralogical Association, Commission on New Minerals and Mineral Names. Can. Min. 1997, 35, 219.

57. Anderson, J.L.; Smith, D.R. The effects of temperature and $f_{\mathrm{O} 2}$ on the Al-in-hornblende barometer. Am. Mineral. 1995, 80, 549-559. [CrossRef]

58. Ernst, W.; Liu, J. Experimental phase-equilibrium study of Al-and Ti-contents of calcic amphibole in MORB-A semiquantitative thermobarometer. Am. Mineral. 1998, 83, 952-969. [CrossRef]

59. Johnson, M.C.; Rutherford, M.J. Experimental calibration of the aluminum-in-hornblende geobarometer with application to Long Valley caldera (California) volcanic rocks. Geology 1989, 17, 837-841. [CrossRef]

60. Hollister, L.S.; Grissom, G.C.; Peters, E.K.; Stowell, H.H.; Sisson, V.B. Confirmation of the empirical correlation of $\mathrm{Al}$ in hornblende with pressure of solidification of calc-alkaline plutons. Am. Mineral. 1987, 72, 231-239.

61. Hammarstrom, J.M.; Zen, E.-A. Aluminum in hornblende: An empirical igneous geobarometer. Am. Mineral. 1986, 71, 1297-1313. 
62. Schmidt, M.W. Amphibole composition in tonalite as a function of pressure: An experimental calibration of the Al-in-hornblende barometer. Contrib. Mineral. Petrol. 1992, 110, 304-310. [CrossRef]

63. Otten, M.T. The origin of brown hornblende in the Artfjället gabbro and dolerites. Contrib. Mineral. Pet. 1984, 86, 189-199. [CrossRef]

64. Helz, R.T. Phase Relations of Basalts in their Melting Range at $\mathrm{P}_{\mathrm{H} 2 \mathrm{O}}=5 \mathrm{~kb}$ as a Function of Oxygen Fugacity1: Part I. Mafic Phases. J. Petrol. 1973, 14, 249-302. [CrossRef]

65. Watson, E.B.; Wark, D.A.; Thomas, J.B. Crystallization thermometers for zircon and rutile. Contrib Miner. Petrol. 2006, 151, 413-433. [CrossRef]

66. Artemieva, I.M.; Shulgin, A. Making and altering the crust: A global perspective on crustal structure and evolution. Earth Planet Sci. Lett. 2019, 512, 8-16. [CrossRef]

67. Leat, P.T.; Larter, R.D.; Larter, R.D.; Leat, P.T. Intra-oceanic subduction systems: Introduction. In Intra-Oceanic Subduction Systems: Tectonic and Magmatic Processes; Geological Society of London: London, UK, 2003; pp. 1-17.

68. Shi, R.D.; Yang, J.S.; Xu, Z.Q.; Qi, X.X. The Bangong Lake ophiolite (NW Tibet) and its bearing on the tectonic evolution of the Bangong-Nujiang suture zone. J. Asian Earth Sci. 2008, 32, 438-457. [CrossRef]

69. White, W.M.; Klein, E.M. 4.13-Composition of the Oceanic Crust. In Treatise on Geochemistry, 2nd ed.; Holland, H.D., Turekian, K.K., Eds.; Elsevier: Oxford, UK, 2014; pp. 457-496. [CrossRef]

70. Takahashi, N.; Yamashita, M.; Kodaira, S.; Miura, S.; Sato, T.; No, T.; Takizawa, K.; Tatsumi, Y.; Kaneda, Y. Rifting Structure of Central Izu-Ogasawara (Bonin) Arc Crust: Results of Seismic Crustal Imaging. In Accretionary Prisms and Convergent Margin Tectonics in the Northwest Pacific Basin; Ogawa, Y., Anma, R., Dilek, Y., Eds.; Springer: Dordrecht, The Netherlands, 2011; pp. 75-95. [CrossRef]

71. Takahashi, N.; Kodaira, S.; Klemperer, S.L.; Tatsumi, Y.; Kaneda, Y.; Suyehiro, K. Crustal structure and evolution of the Mariana intra-oceanic island arc. Geology 2007, 35, 203-206. [CrossRef]

72. Kodaira, S.; Sato, T.; Takahashi, N.; Miura, S.; Tamura, Y.; Tatsumi, Y.; Kaneda, Y. New seismological constraints on growth of continental crust in the Izu-Bonin intra-oceanic arc. Geology 2007, 35, 1031-1034. [CrossRef]

73. Kodaira, S.; Sato, T.; Takahashi, N.; Ito, A.; Tamura, Y.; Tatsumi, Y.; Kaneda, Y. Seismological evidence for variable growth of crust along the Izu intraoceanic arc. J. Geophy. Res. Solid Earth 2007, 112. [CrossRef]

74. Allen, R.W.; Collier, J.S.; Stewart, A.G.; Henstock, T.; Goes, S.; Rietbrock, A.; Team, T.V. The role of arc migration in the development of the Lesser Antilles: A new tectonic model for the Cenozoic evolution of the eastern Caribbean. Geology 2019, 47, 891-895. [CrossRef]

75. Kopp, H.; Weinzierl, W.; Becel, A.; Charvis, P.; Evain, M.; Flueh, E.R.; Gailler, A.; Galve, A.; Hirn, A.; Kandilarov, A.; et al. Deep structure of the central Lesser Antilles Island Arc: Relevance for the formation of continental crust. Earth Planet Sci. Lett. 2011, 304, 121-134. [CrossRef]

76. Macdonald, R.; Hawkesworth, C.J.; Heath, E. The Lesser Antilles volcanic chain: A study in arc magmatism. Earth Sci. Rev. 2000, 49, 1-76. [CrossRef]

77. Bassett, D.; Kopp, H.; Sutherland, R.; Henrys, S.; Watts, A.B.; Timm, C.; Scherwath, M.; Grevemeyer, I.; Ronde, C.E.J. Crustal structure of the Kermadec arc from MANGO seismic refraction profiles. J. Geophy. Res. Solid Earth 2016, 121, 7514-7546. [CrossRef]

78. Contreras-Reyes, E.; Grevemeyer, I.; Watts, A.B.; Flueh, E.R.; Peirce, C.; Moeller, S.; Papenberg, C. Deep seismic structure of the Tonga subduction zone: Implications for mantle hydration, tectonic erosion, and arc magmatism. J. Geophys. Res. 2011, 116. [CrossRef]

79. Turner, S.; Hawkesworth, C. Constraints on flux rates and mantle dynamics beneath island arcs from Tonga-Kermadec lava geochemistry. Nature 1997, 389, 568-573. [CrossRef]

80. Janiszewski, H.A.; Abers, G.A.; Shillington, D.J.; Calkins, J.A. Crustal structure along the Aleutian island arc: New insights from receiver functions constrained by active-source data. Geochem. Geophy. Geosys. 2013, 14, 2977-2992. [CrossRef]

81. Fliedner, M.M.; Klemperer, S.L. Crustal structure transition from oceanic arc to continental arc, eastern Aleutian Islands and Alaska Peninsula. Earth Planet Sci. Lett. 2000, 179, 567-579. [CrossRef]

82. Steven Holbrook, W.; Lizarralde, D.; McGeary, S.; Bangs, N.; Diebold, J. Structure and composition of the Aleutian island arc and implications for continental crustal growth. Geology 1999, 27, 31-34. [CrossRef] 
83. Huang, Q.-T.; Liu, W.-L.; Xia, B.; Cai, Z.-R.; Chen, W.-Y.; Li, J.-F.; Yin, Z.-X. Petrogenesis of the Majiari ophiolite (western Tibet, China): Implications for intra-oceanic subduction in the Bangong-Nujiang Tethys. J. Asian Earth Sci. 2017, 146, 337-351. [CrossRef]

84. Liu, T.; Zhai, Q.-G.; Wang, J.; Bao, P.-S.; Qiangba, Z.; Tang, S.-H.; Tang, Y. Tectonic significance of the Dongqiao ophiolite in the north-central Tibetan plateau: Evidence from zircon dating, petrological, geochemical and Sr-Nd-Hf isotopic characterization. J. Asian Earth Sci. 2016, 116, 139-154. [CrossRef]

85. Zhong, Y.; Xia, B.; Liu, W.-L.; Yin, Z.-X.; Hu, X.-C.; Huang, W. Geochronology, petrogenesis and tectonic implications of the Jurassic Namco-Renco ophiolites, Tibet. Int. Geol. Rev. 2015, 57, 508-528. [CrossRef]

(C) 2019 by the authors. Licensee MDPI, Basel, Switzerland. This article is an open access article distributed under the terms and conditions of the Creative Commons Attribution (CC BY) license (http://creativecommons.org/licenses/by/4.0/). 